\title{
Molecules as tracers of galaxy evolution: an EMIR survey
}

\section{Presentation of the data and first results ${ }^{\star}$}

\author{
F. Costagliola ${ }^{1, \star \star}$, S. Aalto ${ }^{1, \star \star \star}$, M. I. Rodriguez ${ }^{2}$, S. Muller ${ }^{1}$, H. W. W. Spoon ${ }^{3}$, S. Martín ${ }^{4}$, M. A. Peréz-Torres ${ }^{2}$, \\ A. Alberdi ${ }^{2}$, J. E. Lindberg ${ }^{1,5}$, F. Batejat ${ }^{1}$, E. Jütte ${ }^{6}$, P. van der Werf ${ }^{7,8}$, and F. Lahuis ${ }^{7,9}$
}

1 Department of Earth and Space Sciences, Chalmers University of Technology, Onsala Space Observatory, 43992 Onsala, Sweden e-mail: francesco.costagliola@chalmers.se

2 Instituto de Astrofísica de Andalucía (IAA-CSIC), PO Box 3004, 18080 Granada, Spain

3 Cornell University, Astronomy Department, Ithaca, NY 14853, USA

4 European Southern Observarory, Alonso de Córdova 3107, Vitacura, Casilla 19001, Santiago 19, Chile

5 Centre for Star and Planet Formation, Natural History Museum of Denmark, University of Copenhagen, Øster Voldgade 5-7, 1350 København K, Denmark

6 Astronomisches Institut Ruhr-Universitaet Bochum, Universitaetsstr. 150, 44780 Bochum, Germany

7 Leiden Observatory, Leiden University, 2300 RA, Leiden, The Netherlands

8 Institute for Astronomy, University of Edinburgh, Royal Observatory, Blackford Hill, Edinburgh EH9 3HJ, UK

9 SRON Netherlands Institute for Space Research, PO Box 800, 9700 AV, Groningen, The Netherlands

Received 23 August 2010 / Accepted 23 December 2010

\section{ABSTRACT}

\begin{abstract}
Aims. We investigate the molecular gas properties of a sample of 23 galaxies in order to find and test chemical signatures of galaxy evolution and to compare them to IR evolutionary tracers.

Methods. Observation at $3 \mathrm{~mm}$ wavelengths were obtained with the EMIR broadband receiver, mounted on the IRAM $30 \mathrm{~m}$ telescope on Pico Veleta, Spain. We compare the emission of the main molecular species with existing models of chemical evolution by means of line intensity ratios diagrams and principal component analysis.

Results. We detect molecular emission in 19 galaxies in two $8 \mathrm{GHz}$-wide bands centred at 88 and $112 \mathrm{GHz}$. The main detected molecules are $\mathrm{CO},{ }^{13} \mathrm{CO}, \mathrm{HCN}, \mathrm{HNC}, \mathrm{HCO}^{+}, \mathrm{CN}$, and $\mathrm{C}_{2} \mathrm{H}$. We also detect $\mathrm{HC}_{3} \mathrm{~N} J=10-9$ in the galaxies IRAS 17208, IC 860, NGC 4418, NGC 7771, and NGC 1068. The only $\mathrm{HC}_{3} \mathrm{~N}$ detections are in objects with $\mathrm{HCO}^{+} / \mathrm{HCN}<1$. Galaxies with the highest $\mathrm{HC}_{3} \mathrm{~N} / \mathrm{HCN}$ ratios have warm IRAS colours $(60 / 100 \mu \mathrm{m}>0.8)$. The brightest $\mathrm{HC}_{3} \mathrm{~N}$ emission is found in IC 860 , where we also detect the molecule in its vibrationally excited state. We find low $\mathrm{HNC} / \mathrm{HCN}$ line ratios $(<0.5)$, that cannot be explained by existing PDR or XDR chemical models. The intensities of $\mathrm{HCO}+$ and $\mathrm{HNC}$ appear anti-correlated. No correlation is found between the $\mathrm{HNC} / \mathrm{HCN}$ line ratio and dust temperature. All HNC-bright objects are either luminous IR galaxies (LIRG) or Seyferts. Galaxies with bright polycyclic aromatic hydrocarbons $(\mathrm{PAH})$ emission show low $\mathrm{HNC} / \mathrm{HCO}^{+}$ratios. The $\mathrm{CO} /{ }^{13} \mathrm{CO}$ ratio is positively correlated with the dust temperature and is generally higher than in our galaxy. The emission of $\mathrm{CN}$ and $\mathrm{C}^{18} \mathrm{O}$ is correlated.

Conclusions. Bright $\mathrm{HC}_{3} \mathrm{~N}$ emission in $\mathrm{HCO}^{+}$-faint objects may imply that these are not dominated by $\mathrm{X}$-ray chemistry. Thus the $\mathrm{HCN} / \mathrm{HCO}^{+}$line ratio is not, by itself, a reliable tracer of XDRs. Bright $\mathrm{HC}_{3} \mathrm{~N}$ and faint $\mathrm{HCO}^{+}$could be signatures of embedded starformation, instead of AGN activity. Mechanical heating caused by supernova explosions may be responsible for the low $\mathrm{HNC} / \mathrm{HCN}$ and high $\mathrm{HCO}^{+} / \mathrm{HCN}$ ratios in some starbursts. We cannot exclude, however, that the discussed trends are largely caused by optical depth effects or excitation. Chemical models alone cannot explain all properties of the observed molecular emission. Better constraints to the gas spacial distribution and excitation are needed to distinguish abundance and excitation effects.
\end{abstract}

Key words. evolution - ISM: molecules - galaxies: evolution - galaxies: ISM - galaxies: active

\section{Introduction}

Luminous infrared galaxies (LIRGs) radiate most of their luminosity $\left(L_{\mathrm{IR}}>10^{11} L_{\odot}\right)$ as dust thermal emission in the infrared and have been studied at almost all wavelenghts (Sanders $\&$ Mirabel 1996). However, the nature of the power source is still unclear when the inner region of the LIRG is obscured by dust. The high central IR surface brightness implies that this power source can be either an embedded compact starburst or

\footnotetext{
* Appendices are only available in electronic form http://www . aanda.org

$\star \star$ F.C. wishes to thank the EU ESTRELA programme for support.

$\star \star \star$ S.A. wishes to thank the Swedish Research Council for grant support.
}

an enshrouded AGN - or a combination of both. The evolution of the activity and the connection between AGN and starburst are still not well understood and must be further explored. At high redshift, LIRGs dominate the cosmic infrared background and, by assuming that they are powered by starburts, we can use these galaxies to trace the dust-obscured star-formation rate, the dust content and the metallicity in the early Universe (Barger et al. 1999). The most well known techniques to distinguish between AGN and star-powered galaxies rely on the observation of emission lines in the optical (e.g., Veilleux \& Osterbrock 1987). Over the last decade several other diagnostic diagrams, based on IR spectra, have been proposed to quantify the contribution of star-formation and AGN activity to the infrared luminosities of LIRGs (Genzel et al. 1998; Lutz et al. 1998; 
Spoon et al. 2007). In the $\mathrm{mm}$ and sub-mm there have been attempts as well to classify the activity of galaxies via diagnostic diagrams, of which the most well known is the $\mathrm{HCN} / \mathrm{HCO}^{+}$ line ratio plot of Kohno et al. (2001) and Imanishi et al. (2004). A multi-transition study of the $\mathrm{HCN} / \mathrm{HCO}^{+}$ratio in Seyfert and starburst galaxies has also been reported by Krips et al. (2008). These authors find an underluminosity of $\mathrm{HCO}^{+}$in some AGN-dominated cores, which they suggested to be owing to an underabundance of $\mathrm{HCO}^{+}$caused by the X-ray-dominated chemistry induced by the AGN (Maloney et al. 1996). This latter interpretation has been disputed since it does not agree with recent models of X-ray-dominated regions (XDRs) (Meijerink $\&$ Spaans 2005), which instead show an enhancement of $\mathrm{HCO}^{+}$ abundances caused by an increase in ionization. Other interpretations in terms of starburst evolution have been put forward as an alternative (e.g., Baan et al. 2008). Graciá-Carpio et al. (2006) also found that ULIRGs $\left(L_{\mathrm{IR}}>10^{12} L_{\odot}\right)$ in general seem to have lower $\mathrm{HCO}^{+} 1-0$ luminosities with regards to $\mathrm{HCN}-$ compared with more moderate LIRGs. The HCN/CN 1-0 and $\mathrm{HCN} / \mathrm{HNC} 1-0$ line ratios have been used as well to help interpret a galaxy's position in an evolutionary scheme (Aalto et al. 2002; Baan et al. 2008). For the brightest nearby galaxies, a scheme based on the $\mathrm{HNCO} / \mathrm{CS}$ ratio has been proposed (Martín et al. 2009). Moreover, prominent sources such as NGC 253 and IC 342 allow the detection of rarer species that can be used in turn to help identifying and even resolving the dominant activity (e.g., Meier \& Turner 2005; Martín et al. 2006).

Line ratios need to be very accurately measured if we want them to be sensitive tracers of molecular properties. The new EMIR receiver, mounted in 2009 on the IRAM 30 m telescope in Spain, offers the opportunity of achieving this high accuracy. The available bandwidth of nearly $8 \mathrm{GHz}$ at $3 \mathrm{~mm}$ allows us to fit many key molecular lines in the same band, therefore eliminating the uncertainties owing to relative calibration and pointing errors that affect most single-dish observations. We present a survey of molecular lines for a sample of 23 galaxies observed with EMIR in the period June-November 2009. In Sect. 3 we report the details about the observations and source selection. In Sect. 4 we present the results. In Sect. 5 the line ratio diagrams for $\mathrm{HCN}, \mathrm{HNC}, \mathrm{HCO}^{+}$, and $\mathrm{HC}_{3} \mathrm{~N}$ are presented and discussed. The molecular emission is also compared with far-infrared (FIR) colours and polycyclic aromatic hydrocarbons (PAH) emission. In Sect. 6 we present our conclusions and an outlook. The spectra of all observed sources and the tables summarizing the line parameters of the detected species can be found in the Appendix.

\section{Source selection}

The first diagnostic diagram to take into account the effects of strong obscuration of the nuclear power source was presented by Spoon et al. (2007), using the equivalent width of the $6.2 \mu \mathrm{m}$ PAH emission feature and the strength of the $9.7 \mu \mathrm{m}$ silicate absorption (see Fig. 1).

Based on the position in the diagram, galaxies are put into nine classes, ranging from continuum-dominated AGN hot dust spectra $(1 A)$ to PAH-dominated starburst spectra $(1 C)$ to absorption-dominated spectra of deeply obscured galactic nuclei $(3 A)$.

Spoon et al. (2007) find that galaxies are systematically distributed along two distinct branches: one horizontal sequence at low silicate depths, ranging from AGN to starburst-dominated spectra, and one diagonal sequence at higher silicate strength, ranging from obscured nuclei to pure-starburst objects. The separation into two branches likely reflects fundamental differences

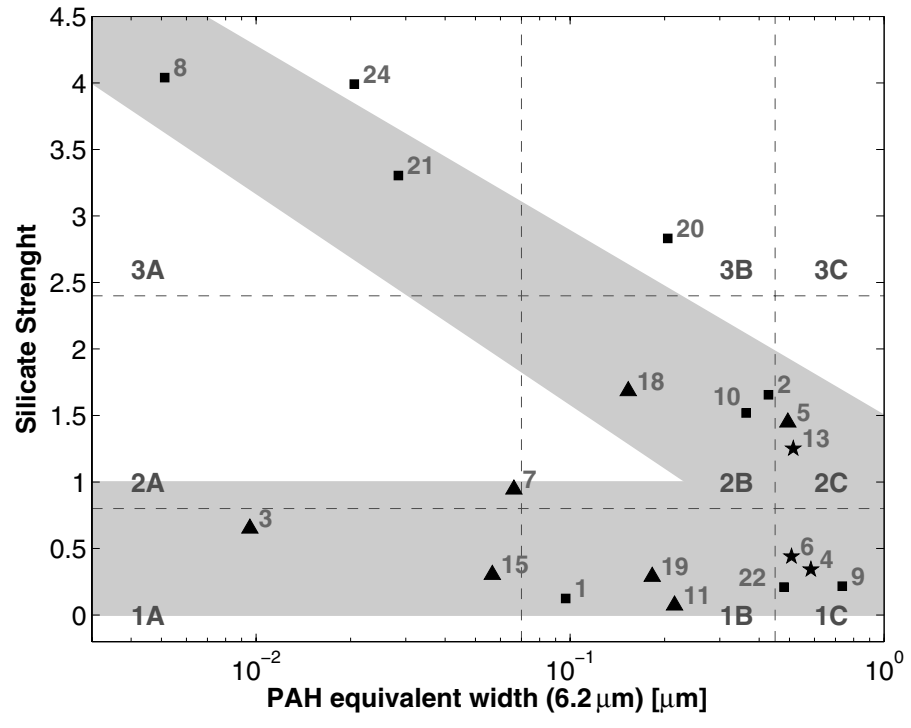

Fig. 1. Position of the observed galaxies on the mid-infrared diagnostic diagram from Spoon et al. (2007). Galaxies are divided into nine classes $(1 A-3 C)$, depending on their spectral properties and position on the graph. These classes range from PAH-dominated spectra $(1 C)$ to continuum-dominated spectra with faint PAH emission $(1 A)$ and obscured galaxies with deep silicate absorption (3A). Galaxy types are distinguished by their plotting symbol: squares: LIRGs. Stars: starburst galaxies. Triangles: seyfert galaxies. The numbers indicate different galaxies, as explained in Table 1 . The shadowed area corresponds to the two main sequences discussed by Spoon et al. (2007). An increase of the PAH equivalent width indicates an increase of the starburst contribution to the emission, while AGN spectra are continuum-dominated. See Sect. 2 for further discussion.

in the dust geometry in the two sets of sources. Spectra of luminous infrared galaxies are found along the full length of both branches, reflecting the diverse nature of the LIRG family. In this work, the term $L I R G$ broadly refers to objects which emit most of their energy in the IR. Thus a galaxy classified as LIRG may be a starburst, an AGN, or both, but the dust obscuration hinders a clear classification. We do not explicitly distinguish between LIRGs $\left(L_{\mathrm{IR}}>10^{11} L_{\odot}\right)$ and ULIRGs $\left(L_{\mathrm{IR}}>10^{12} L_{\odot}\right)$. However, IR luminosities are reported in Table 1 for reference. Objects with both LIRG and AGN signatures are labelled as $A, L$ in Table 1 and appear as AGNs (triangles) in the graphs.

Spoon et al. (2007) interpret the observed distribution as a possible evolutionary effect, with sources moving from the diagonal to the horizontal branch as the dust distribution evolves from a uniform to a clumpy geometry. The underpopulated $2 \mathrm{~A}$ class implies either that this transition for LIRGs is very rapid, or that LIRGs mostly evolve into unobscured starbursts.

We aim to compare this mid-IR evolution scheme with $\mathrm{mm}$ molecular observations. Our targets were mainly selected from the sample of Spoon et al. (2007), plus a few interesting objects for which we had PAH and silicate mid-IR data. The source selection criteria were the following:

- uniform coverage of the most significant classes in the diagnostic diagram of Spoon et al. (2007);

- uniform representation of different galaxy types (Seyferts, starbursts, LIRGs);

- source visibility at IRAM site.

The resulting sample is composed of 23 galaxies, whose main properties are listed in Table 1. All targets were observed in the 
Table 1. Line ratios and general properties of the observed galaxies.

\begin{tabular}{|c|c|c|c|c|c|c|c|c|c|c|c|c|}
\hline Galaxy & $\begin{array}{c}\text { RA } \\
{[\mathrm{h}: \mathrm{m}: \mathrm{s}]}\end{array}$ & $\begin{array}{c}\text { Dec } \\
{\left[{ }^{\circ}::^{\prime \prime}\right]}\end{array}$ & $\begin{array}{c}V_{\text {Helio }} \\
{\left[\mathrm{km} \mathrm{s}^{-1}\right]}\end{array}$ & $\frac{\mathrm{HCO}^{+}(1-0)}{\mathrm{HCN}(1-0)}$ & $\frac{\overline{\mathrm{HNC}(1-0)}}{\mathrm{HCN}(1-0)}$ & $\frac{\mathrm{HC}_{3} \mathrm{~N}(10-9)}{\mathrm{HCN}(1-0)}$ & 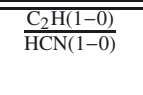 & $\frac{\mathrm{CO}(1-0)}{\frac{13}{13 \mathrm{CO}(1-0)}}$ & $\overline{\log \left(L_{\mathrm{IR}} / L_{\odot}\right)}$ & "Class & Type & Num. \\
\hline IRAS 17208 & $17: 23: 21.9$ & $-00: 17: 01$ & 12834 & $0.78(0.12)$ & $0.77(0.14)$ & $0.31(0.07)$ & $0.42(0.10)$ & - & 12.35 & 1B & $\mathrm{L}$ & 1 \\
\hline IC 860 & $13: 15: 03.5$ & $+24: 37: 08$ & 3347 & $0.62(0.16)$ & $0.62(0.16)$ & $0.42(0.13)$ & $1.04(0.29)$ & $18.96(3.83)$ & 11.14 & $2 \mathrm{~B}$ & $\mathrm{~L}$ & 2 \\
\hline Mrk 231 & $12: 56: 14.2$ & $+56: 52: 25$ & 12642 & $0.56(0.08)$ & $0.38(0.06)$ & $<0.07$ & $0.29(0.12)$ & - & 12.37 & $1 \mathrm{~A}$ & $\mathrm{~A}, \mathrm{~L}$ & 3 \\
\hline NGC 1614 & 04:33:59.8 & $-08: 34: 44$ & 4778 & $1.83(0.37)$ & $0.33(0.15)$ & $<0.35$ & $1.00(0.33)$ & $28.81(2.10)$ & 11.43 & $1 \mathrm{C}$ & S & 4 \\
\hline NGC 3079 & 10:01:57.8 & $+55: 40: 47$ & 1116 & $1.12(0.11)$ & $0.27(0.05)$ & $<0.06$ & $0.54(0.09)$ & $17.10(1.16)$ & 10.65 & $2 \mathrm{C}$ & A & 5 \\
\hline NGC 4194 & $12: 14: 09.5$ & $+54: 31: 37$ & 2501 & $1.32(0.35)$ & $0.53(0.23)$ & $<0.20$ & $0.98(0.36)$ & $19.10(1.90)$ & 10.93 & $1 \mathrm{C}$ & S & 6 \\
\hline NGC 4388 & $12: 25: 46.7$ & $+12: 39: 44$ & 2524 & $1.38(0.40)$ & $0.62(0.24)$ & $<0.35$ & $0.81(0.52)$ & - & 9.66 & $2 \mathrm{~A}$ & A & 7 \\
\hline NGC 4418 & $12: 26: 54.6$ & $-00: 52: 39$ & 2110 & $0.59(0.10)$ & $0.47(0.09)$ & $0.37(0.09)$ & $0.64(0.18)$ & - & 11.00 & $3 \mathrm{~A}$ & $\mathrm{~L}$ & 8 \\
\hline NGC 6090 & $16: 11: 40.7$ & $+52: 27: 24$ & 8785 & $1.67(0.44)$ & $0.25(0.17)$ & $<0.18$ & $0.46(0.20)$ & - & 11.34 & $1 \mathrm{C}$ & $\mathrm{L}$ & 9 \\
\hline NGC 6240 & $16: 52: 58.9$ & $+02: 24: 03$ & 7339 & $1.63(0.14)$ & $0.20(0.06)$ & $<0.09$ & $0.36(0.08)$ & $28.79(2.99)$ & 11.69 & $2 \mathrm{~B}$ & $\mathrm{~L}$ & 10 \\
\hline NGC 7469 & $23: 03: 15.6$ & $+08: 52: 26$ & 4892 & $1.12(0.11)$ & $0.55(0.08)$ & $<0.07$ & $0.75(0.24)$ & $20.80(0.47)$ & 11.41 & $1 \mathrm{~B}$ & A & 11 \\
\hline NGC 7771 & $23: 51: 24.9$ & $+20: 06: 43$ & 4277 & $0.94(0.09)$ & $0.44(0.07)$ & $0.06(0.04)$ & $0.32(0.06)$ & $13.61(0.52)$ & 11.24 & - & $\mathrm{L}$ & 12 \\
\hline NGC 660 & $01: 43: 02.4$ & $+13: 38: 42$ & 850 & $1.04(0.09)$ & $0.52(0.06)$ & $<0.07$ & $0.45(0.08)$ & $16.45(0.49)$ & 10.40 & $2 \mathrm{C}$ & S & 13 \\
\hline NGC 3556 & $11: 11: 31.0$ & $+55: 40: 27$ & 699 & $1.57(0.37)$ & $0.37(0.24)$ & $<0.27$ & $1.56(0.37)$ & $12.50(0.31)$ & 10.00 & - & S & 14 \\
\hline NGC 1068 & $02: 42: 40.7$ & $-00: 00: 48$ & 1137 & $0.67(0.03)$ & $0.40(0.03)$ & $0.04(0.02)$ & $0.37(0.09)$ & - & 10.89 & $1 \mathrm{~A}$ & A & 15 \\
\hline NGC 7674 & $23: 27: 56.7$ & $+08: 46: 45$ & 8671 & - & - & - & - & $14.46(1.18)$ & 11.50 & - & A & 16 \\
\hline UGC 2866 & $03: 50: 14.9$ & $+70: 05: 41$ & 1232 & $1.46(0.20)$ & $0.51(0.13)$ & $<0.14$ & $0.88(0.17)$ & $20.68(0.80)$ & 10.69 & - & S & 17 \\
\hline UGC 5101 & $09: 35: 51.6$ & $+61: 21: 11$ & 11802 & $0.36(0.20)$ & $0.82(0.28)$ & $<0.28$ & $0.89(0.37)$ & $>8.63$ & 11.87 & $2 \mathrm{~B}$ & $\mathrm{~A}, \mathrm{~L}$ & 18 \\
\hline NGC 2273 & 06:50:08.6 & $+60: 50: 45$ & 1840 & $1.05(0.37)$ & $1.09(0.38)$ & $<0.89$ & $<0.89$ & - & 10.11 & 1B & A & 19 \\
\hline Arp 220 & $15: 34: 57.2$ & $+23: 30: 09$ & 5382 & $0.47(0.07)$ & $0.49(0.12)$ & $0.19(0.06)$ & - & - & 12.15 & $3 \mathrm{~B}$ & $\mathrm{~L}$ & 20 \\
\hline IRAS 15250 & $15: 26: 59.4$ & $+35: 58: 38$ & 16535 & - & - & - & - & - & 12.02 & $3 \mathrm{~A}$ & $\mathrm{~L}$ & 21 \\
\hline NGC 1140 & $02: 54: 33.6$ & $-10: 01: 40$ & 1501 & - & - & - & - & - & 9.50 & $1 \mathrm{C}$ & $S$ & 22 \\
\hline NGC 1056 & $02: 42: 48.3$ & $+28: 34: 27$ & 1545 & - & - & - & - & - & 9.50 & - & S & 23 \\
\hline NGC 1377 & 03:36:39.1 & $-20: 54: 07$ & 1792 & - & - & - & - & - & 9.63 & $3 \mathrm{~A}$ & $\mathrm{O}$ & 24 \\
\hline
\end{tabular}

Notes. The mid-IR Class column refers to the classification by Spoon et al. (2007), based on the PAH equivalent width and silicate absorption in the mid-infrared. The column Type summarizes the properties of the object, distinguishing between starbursts $(S)$, Seyferts $(A)$, and LIRGs $(L)$. The galaxy NGC 1377 is classified as obscured $(O)$, because it emits mostly in the IR, but its luminosity is not high enough to be classified as LIRG. For a discussion about source types see Sect. 2. In Figs. 1, 4 and 5, the sources are labelled with the numbers reported in column Num.

$88 \mathrm{GHz}$ band, but because of time constraints, only 12 were observed in the $112 \mathrm{GHz}$ band.

\section{Observations}

The observations were obtained in June-November 2009 with the IRAM $30 \mathrm{~m}$ telescope on Pico Veleta, Spain. The $8 \mathrm{GHz}$ band of the EMIR receiver was centred on two different tunings, at 88.675 and $112.15 \mathrm{GHz}$. These frequencies were chosen in order to accommodate as many potentially strong lines as possible in the same band. A list of the transitions of the most important molecular gas tracers is given in Table 2, together with beam efficiencies $\left(\eta_{\mathrm{mb}}\right)$ and beam sizes (HPBW) for the two observed bands. Observations were performed in dual polarization, each covering $8 \mathrm{GHz}$, which nicely fitted the $4 \times 4 \mathrm{GHz}$ backend bottleneck. The E090 frontend was connected to the low-resolution WILMA autocorrelator, which is capable of processing the large input bandwidth with a resolution of about $2 \mathrm{MHz}(\simeq 7$ and $5 \mathrm{~km} \mathrm{~s}^{-1}$ at $88 \mathrm{GHz}$ and $112 \mathrm{GHz}$, respectively). Observations were performed in wobbler switching mode, with a throw of 60$120^{\prime \prime}$ (depending on source size), in order to maximize baseline quality.

The pointing model was checked against bright, nearby calibrators for every source, and every two hours for long integrations. Calibration scans on the standard two load system were taken every $5 \mathrm{~min}$. The observing conditions were optimal, with characteristic system temperatures of 100 and $150 \mathrm{~K}$ for the
Table 2. Properties of the two observed bands.

\begin{tabular}{|c|c|c|c|c|c|}
\hline Line & $\begin{array}{c}\text { HPBW } \\
{\left[{ }^{\prime \prime}\right]}\end{array}$ & $\eta_{\mathrm{mb}}$ & $\begin{array}{c}v_{0} \\
{[\mathrm{GHz}]}\end{array}$ & $\begin{array}{l}E_{\text {up }} \\
{[\mathrm{K}]}\end{array}$ & $\begin{array}{c}n_{\mathrm{c}}(20 \mathrm{~K}) \\
{\left[\mathrm{cm}^{-3}\right]}\end{array}$ \\
\hline $88 \mathrm{GHz}$ Band & 29 & 0.81 & & & \\
\hline $\mathrm{SiO}(2-1)$ & $\prime \prime$ & " & 86.847 & 6.25 & $1 \times 10^{5}$ \\
\hline $\mathrm{C}_{2} \mathrm{H}(1-0)$ & " & " & 87.316 & 4.19 & $1 \times 10^{5}$ \\
\hline $\mathrm{HCN}(1-0)$ & " & " & 88.633 & 4.25 & $2 \times 10^{5}$ \\
\hline $\mathrm{HCO}^{+}(1-0)$ & " & " & 89.188 & 4.28 & $3 \times 10^{4}$ \\
\hline $\mathrm{HNC}(1-0)$ & " & " & 90.663 & 4.35 & $1 \times 10^{5}$ \\
\hline $\mathrm{HC}_{3} \mathrm{~N}(10-9)$ & $"$ & $"$ & 90.978 & 24.0 & $9 \times 10^{4}$ \\
\hline $112 \mathrm{GHz}$ Band & 22 & 0.78 & & & \\
\hline $\mathrm{HC}_{3} \mathrm{~N}(12-11)$ & " & " & 109.173 & 34.0 & $2 \times 10^{5}$ \\
\hline $\mathrm{C}^{18} \mathrm{O}(1-0)$ & " & " & 109.757 & 5.27 & $4 \times 10^{2}$ \\
\hline${ }^{13} \mathrm{CO}(1-0)$ & " & " & 110.201 & 5.29 & $4 \times 10^{2}$ \\
\hline $\mathrm{CN}(1-0) J=1 / 2-1 / 2$ & " & " & 113.191 & 5.43 & $2 \times 10^{6}$ \\
\hline $\mathrm{CO}(1-0)$ & " & " & 115.271 & 5.53 & $4 \times 10^{2}$ \\
\hline
\end{tabular}

Notes. Rest frequencies of the brightest lines in each band are shown with the beam sizes (HPBW) and main beam efficiencies $\left(\eta_{\mathrm{mb}}\right)$. Energies of upper level $\left(E_{\text {up }}\right)$ and critical densities at $20 \mathrm{~K}\left(n_{\mathrm{c}}\right)$ were taken from the Leiden atomic and molecular database.

$88 \mathrm{GHz}$ and $112 \mathrm{GHz}$ observations, respectively. This resulted in a rms noise per channel of roughly $0.6 \mathrm{mK}$ at $60 \mathrm{~km} \mathrm{~s}^{-1}$ resolution, for $2 \mathrm{~h}$ of on-source observing time. 

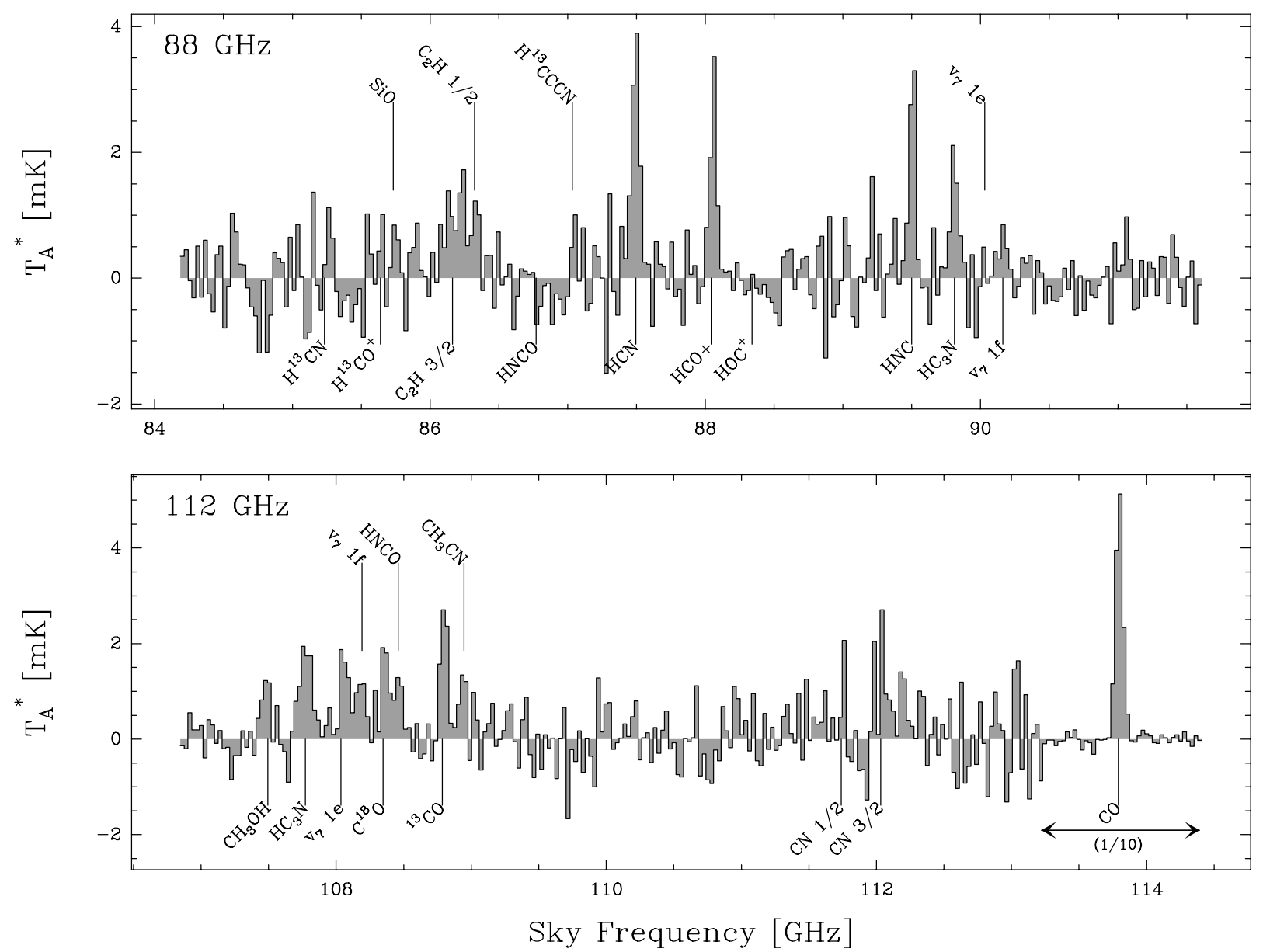

Fig. 2. Spectra observed with EMIR in IC 860 at 88 and $112 \mathrm{GHz}$. The intensity scale is in $T_{\mathrm{A}}^{\star}$, not corrected for main beam efficiency. The region marked with (1/10), around the CO 1-0 line, has been scaled down by a factor 10. The main molecular transitions are labelled regardless of line detection. The $\mathrm{C}_{2} \mathrm{H} 3 / 2$ and $1 / 2$ labels mark the limits of the $\mathrm{C}_{2} \mathrm{H}$ multiplet at $87 \mathrm{GHz}$. Transitions of vibrationally excited $\mathrm{HC}_{3} \mathrm{~N}$ are labelled as $v_{7} 1 e$ and $v_{7} 1 f$. The frequency scale is the observed frequency, not corrected for redshift.

Data were reduced with the CLASS ${ }^{1}$ software. A first order baseline was removed from all spectra, which are shown in Appendix D. The intensity scale in all spectra is in $T_{\mathrm{A}}^{*}$, which is related to the source brightness temperature as

$T_{\mathrm{b}}=\frac{T_{\mathrm{A}}^{*}}{\eta_{\mathrm{mb}}} \times \frac{\theta_{\mathrm{s}}^{2}+\theta_{\mathrm{b}}^{2}}{\theta_{\mathrm{s}}^{2}}$,

with $\theta_{\mathrm{s}}$ and $\theta_{\mathrm{b}}$ the angular sizes of source and beam, respectively. We assume a constant main beam efficiency across both observed bands. The error on the main beam brightness temperature estimate introduced by this assumption is of the order of one percent.

\section{Results}

Rest frequencies were taken from the NIST database Recommended Rest Frequencies for Observed Interstellar Molecular Microwave Transitions ${ }^{2}$. Our line identification takes into account the distortion of the velocity scale caused by the large observed bandwidth. For a discussion of this effect, see Gordon et al. (1992).

\footnotetext{
${ }^{1}$ http://iram.fr/IRAMFR/GILDAS/

2 http://physics.nist.gov/PhysRefData/Micro/Html/ contents.html
}

An example of an EMIR spectrum is shown in Fig. 2, while all the observed spectra are shown in Appendix D. Line intensities were extracted by means of Gaussian fitting and are reported along with other line parameters in Appendix E.

Integrated intensities of the most relevant species were combined to form line intensity ratios, which are listed in Table 1. These were used to produce the diagrams shown in Figs. 4 and 5. We choose to compare intensities of transitions inside the same frequency band to maximize the accuracy of the derived line ratios. The diagrams derived from the two observed bands are described in Sects. 4.1 and 4.2. A principal component analysis of the $88 \mathrm{GHz}$ dataset is discussed in Sect. 4.3.

\subsection{Line ratios at $88 \mathrm{GHz}$}

In plots $a, b$, and $c$ of Fig. 4, we report the line ratios between the first transitions $(J=1-0)$ of $\mathrm{HCN}, \mathrm{HNC}$ and $\mathrm{HCO}^{+}$for all detected galaxies. The different symbols refer to different galaxy types. We note that all non-compact starburst galaxies (stars) are $\mathrm{HCO}^{+}$-luminous, with line ratios $\mathrm{HCO}^{+} / \mathrm{HCN} \geq 1$ (plots $a$, b). Luminous infrared galaxies (squares) and Seyferts (triangles) do not show any strong trend. However, the majority of active galactic nuclei do reside at low $\mathrm{HCO}^{+} / \mathrm{HCN}$ values compared with starbursts.

The $\mathrm{HC}_{3} \mathrm{~N} / \mathrm{HCN}$ line ratio is also reported in the graphs. Circles are drawn around sources where $\mathrm{HC}_{3} \mathrm{~N} J=10-9$ has 

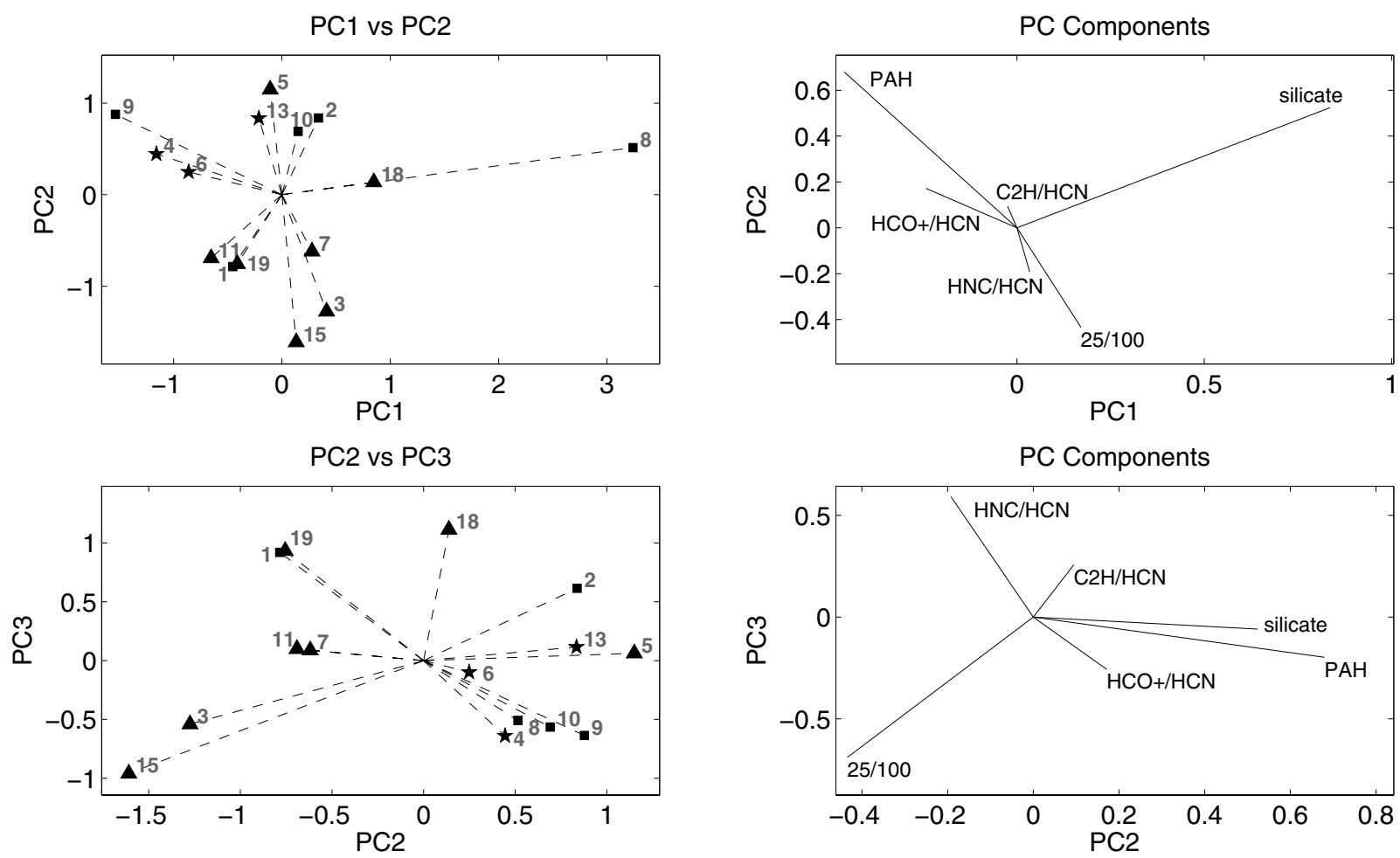

Fig. 3. Results from the principal component analysis. Left: position of the observed galaxies in the PC base $[P C 1, P C 2, P C 3]$. Different symbols refer to starbursts (stars), Seyferts (triangles) and LIRGs (squares). Galaxies with both AGN and LIRG properties are plotted as triangles. Numbers indicate different galaxies, as explained in Table 1. Values for the PAH equivalent widths are taken from Spoon et al. (2007). Infrared fluxes were obtained from the NASA/IPAC Extragalactic Database. Right: projection of the PC components onto the original base of observables. We consider only the first three PCs, since they account for $90 \%$ of the variance and thus contain most of the information in the dataset.

been detected, the diameter of the circle being proportional to the $\mathrm{HC}_{3} \mathrm{~N} / \mathrm{HCN}$ ratio. Evidently all the $\mathrm{HC}_{3} \mathrm{~N}$ detections have $\mathrm{HCO}^{+} / \mathrm{HCN}<1$ and $\mathrm{HNC} / \mathrm{HCN}>0.4$.

Plots $a$ and $c$ show strong correlations. As $\mathrm{HNC} / \mathrm{HCO}^{+}$increases, galaxies move on the graphs towards higher $\mathrm{HNC} / \mathrm{HCN}$ and $\mathrm{HCO}^{+} / \mathrm{HCN}$ ratios. An inverse correlation between $\mathrm{HNC}$ and $\mathrm{HCO}^{+}$line intensities also emerges from plot $b$. Galaxies at $\mathrm{HCO}^{+} / \mathrm{HCN}<1$ present on average a $30 \%$ increase in $\mathrm{HNC} / \mathrm{HCN}$, compared with those at higher $\mathrm{HCO}^{+} / \mathrm{HCN}$ ratios. If we exclude the Seyfert NGC 2273, the higher end of the HNC luminosity distribution (see graphs $b$ and $c$ in Fig. 4) is composed mainly of luminous infrared galaxies.

The general picture emerging from the graphs is that starbursts are characterized by faint $\mathrm{HNC}$ and bright $\mathrm{HCO}^{+}$emission (compared with HCN), while LIRGs mostly occupy the opposite end of the $\mathrm{HNC}-\mathrm{HCO}^{+}$correlation, with high $\mathrm{HNC}$ and low $\mathrm{HCO}^{+}$intensities. All HNC-bright galaxies are either LIRGs or Seyferts. Most of the $\mathrm{HC}_{3} \mathrm{~N}$ detections are LIRGs, the only exception being the Seyfert NGC 1068.

\subsection{Line ratios at $112 \mathrm{GHz}$}

The main detections in the $112 \mathrm{GHz}$ band are the $J=1-0$ emission lines of $\mathrm{CO},{ }^{13} \mathrm{CO}$ and $\mathrm{C}^{18} \mathrm{O}$, and the spin doublet of $\mathrm{CN} 1-0$ $J=3 / 2-1 / 2$ and $J=1 / 2-1 / 2$. These lines are detected in most of the galaxies in our sample, and their integrated intensities are compared in the plots in Fig. 5.

A particularly rich chemistry is found in IC 860, whose spectrum shows bright emission lines of methanol and $\mathrm{HC}_{3} \mathrm{~N}$
$J=12-11$. This detection of $\mathrm{HC}_{3} \mathrm{~N}$ is the only one in the 112 $\mathrm{GHz}$ sample and will be further discussed in Sect. 5.8.

\subsection{Principal component analysis}

Line-ratio diagrams as the ones shown in Sects. 4.1 and 4.2 represent the standard framework for interpreting molecular data and can be easily compared with previous studies (e.g., Baan et al. 2008; Loenen et al. 2008; Baan et al. 2010), as discussed in Sect. 4.4. In this approach, the properties of different galaxy types are compared two at a time to find the observables that best characterize different physical environments. In general, however, the observed quantities are not independent and different environments are best described by a combination of observables. As the number of observables increases, it becomes more and more difficult to interpret multidimensional datasets by means of $2 \mathrm{D}$ sections (i.e., line-ratio diagrams).

To address this complexity, we applied a principal component analysis (PCA) to our data. This is commonly used to reduce the dimensionality of a dataset and is very effective in finding hidden trends that could otherwise be buried in the noise. For an application of a PCA to molecular maps, see, e.g., Ungerechts et al. (1997) and Meier \& Turner (2005).

Each galaxy in our sample is described by a set of $n$ observed quantities (e.g. line ratios, IR properties) that represent an initial base of vectors. The PCA algorithm first computes the covariance matrix of the data along the $n$ directions and finds its eigenvalues and eigenvectors. The eigenvalues are then sorted in descending order, and the corresponding eigenvectors labelled as principal component $(P C) 1,2$, etc. 

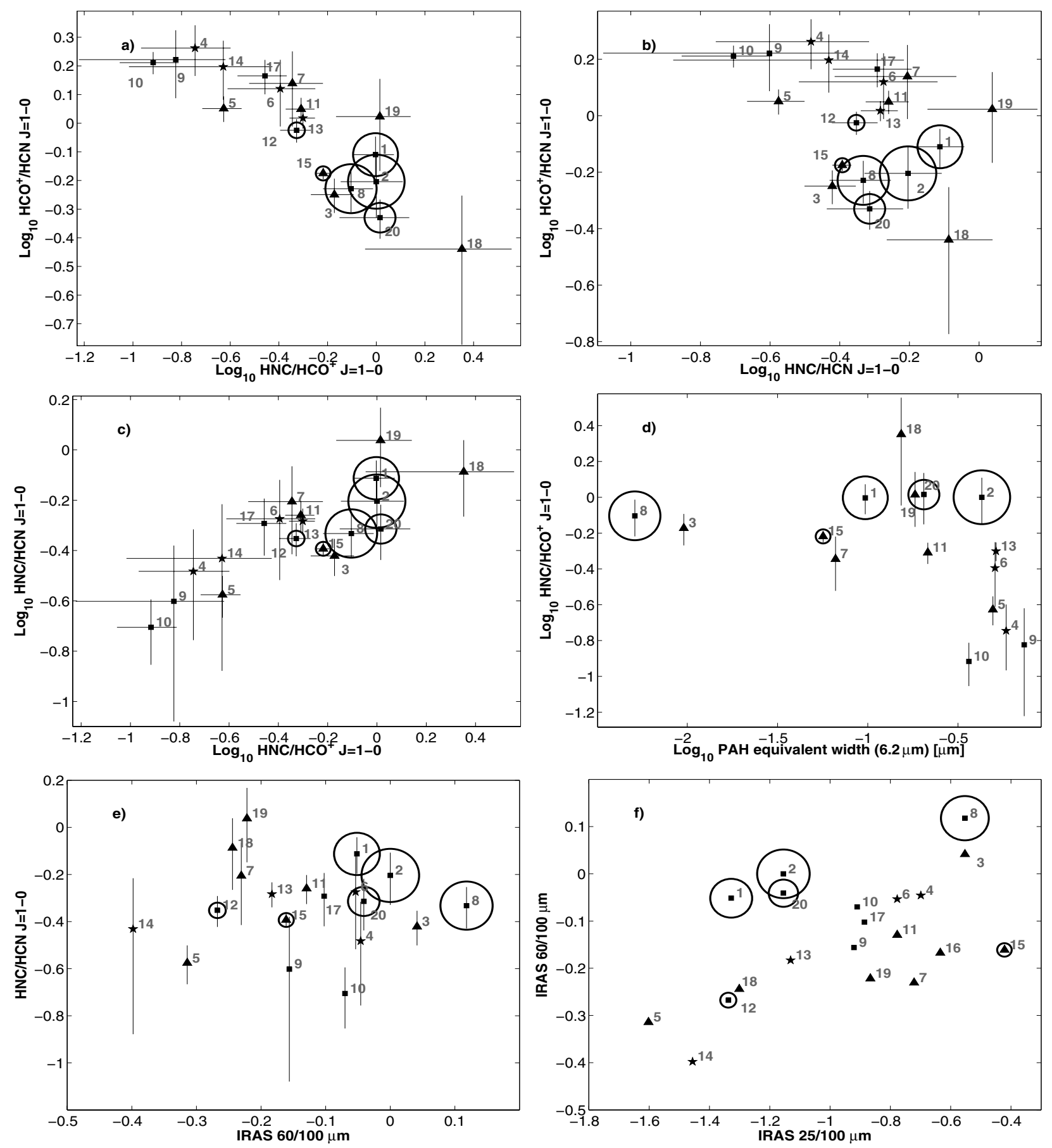

Fig. 4. Diagrams derived from observed line ratios. Symbols and galaxy labelling are the same as in Fig. 3. Galaxies where $\mathrm{HC}_{3} \mathrm{~N}$ was detected are marked with a circle. The diameter of the circle is proportional to the $\mathrm{HC}_{3} \mathrm{~N} 10-9 / \mathrm{HCN} 1-0$ line ratio. Error bars show 1- $\sigma$ uncertainties.

The first principal component (PC 1) is thus the linear combination of the initial galaxy properties along which the dispersion of the data is maximum. PC 2 is the vector, perpendicular to PC 1 , which has the second highest dispersion, and so on for all $n$ PCs. The values of the observed properties for each galaxy are then projected on the new base of PCs.

In our analysis we choose as a base of observables the line intensity ratios $\mathrm{HNC} / \mathrm{HCN}, \mathrm{HCO}^{+} / \mathrm{HCN}$, and $\mathrm{C}_{2} \mathrm{H} / \mathrm{HCN}$ and the IR properties silicate absorption, PAH EW and the ratio of the IRAS fluxes at 25 and $100 \mu \mathrm{m}$. Because our algorithm cannot deal with upper limits, we do not include faint lines, as $\mathrm{HC}_{3} \mathrm{~N}$. In order to maximize the number of galaxies, we limit our analysis to the $88 \mathrm{GHz}$ band, since only a fraction of our sample was observed at $112 \mathrm{GHz}$.

\subsubsection{Results of the PC analysis}

The results of the PCA algorithm are shown in Table 4. Here the projection of the PCs onto the original base are reported along with their contribution to the total dispersion in the dataset. Most of the information is contained in the first three PCs, which account for about $90 \%$ of the total variance. In the following analysis we will thus focus on PC 1,2,3. 


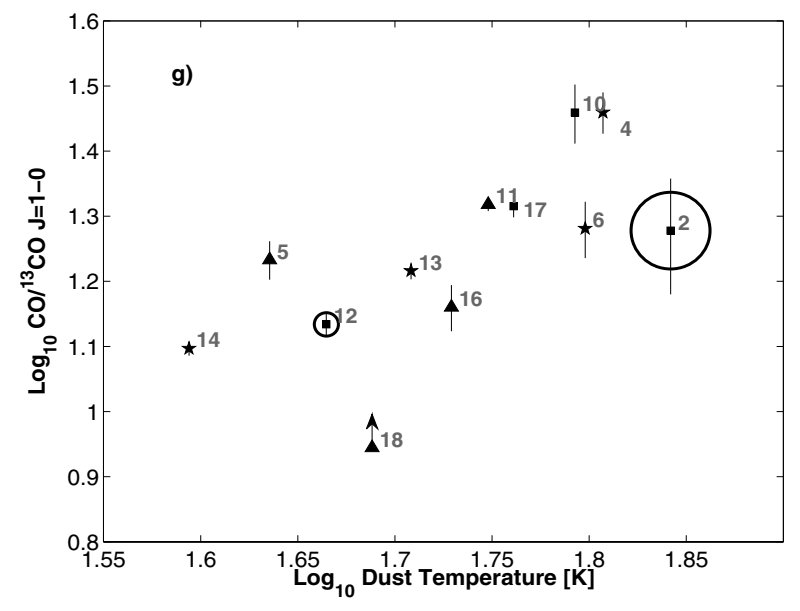

Fig. 5. See caption of Fig. 4 and discussion in Sects. 5.5 and 5.6.

In Fig. 3 right we show the projection of the PCs onto the original base of observables, while in Fig. 3 left we plot the position of the galaxies in the PC base.

The main contribution to the dispersion of the data comes from PAH EW and silicate absorption, which have the highest projections on PC 1. The opposite signs mean that the dispersion along PC1 is mainly caused by galaxies that have low PAH EW and high silicate absorption, or vice versa. The first PC is therefore dominated by the diagonal sequence on the mid-IR diagram of Fig. 1. Galaxies with high values of PC 1 will have high silicate absorption and low PAH EW. This is evident when comparing Figs. 1 and 3 left, where the galaxies NGC 4418 and NGC 6090 (8 and 9 on the graphs) lie at the opposite ends of both PC 1 and the mid-IR sequence.

Among the molecular line intensity ratios the largest contribution to $\mathrm{PC} 1$ is given by $\mathrm{HCO}^{+} / \mathrm{HCN}$. From Fig. 3 left, $\mathrm{HCO}^{+} / \mathrm{HCN}$ appears to be positively correlated with PAH EW (same sign of the projection along PC 1). This agrees with Figs. $4 d$ and B.1 and generally with the trend that will be discussed in Sect. 5.10.

The second principal component (PC 2) is also dominated by IR observables, but with different contributions than the ones derived for PC 1. Silicates and PAH projections are now positively correlated, and the ratio IRAS $25 / 100 \mu \mathrm{m}$ gives a significant contribution to the dispersion. As can be seen in Fig. 3 le ft, PC 2 efficiently separates AGNs from obscured or starburstdominated sources. All AGNs, with the exception of NGC 3079 (number 5 on the graph), have negative PC 2 values, which correspond roughly to classes $1 \mathrm{~A}-1 \mathrm{~B}$ on the mid-IR diagram of Fig. 1. An interesting anti-correlation between PAH EW and IRAS $25 / 100 \mu \mathrm{m}$ flux ratio is also evident in Fig. 3 left. This may be caused by higher dust temperatures in systems with a dominant AGN component.

Most of the molecular information is contained in PC 3, where a substantial fraction of the total dispersion is along the $\mathrm{HNC} / \mathrm{HCN}-\mathrm{HCO}^{+} / \mathrm{HCN}$ direction, while the contribution by $\mathrm{PAH}$ and silicates is almost negligible. The IRAS colour $(25 / 100 \mu \mathrm{m}$ ratio) still plays an important role, with a projection along PC $3(\simeq-0.7)$ slightly higher in absolute value than the one for the $\mathrm{HNC} / \mathrm{HCN}$ line intensity ratio $(\simeq 0.6)$. The opposite sign of these two projections is caused by a slight anti-correlation of the $\mathrm{HNC} / \mathrm{HCN}$ ratio with dust temperature, which also results from the correlation matrix in Table 3 . This trend, however, is not evident in Fig. 4e. The distribution of galaxies along PC 3

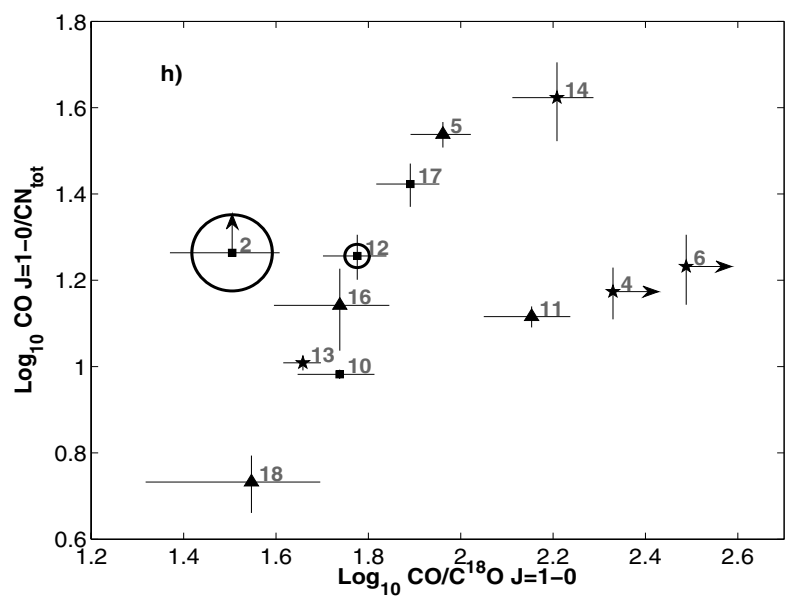

Table 3. Correlation matrix.

\begin{tabular}{lcccccc}
\hline \hline & $\mathrm{HNC} / \mathrm{HCN}$ & $\mathrm{HCO}^{+} / \mathrm{HCN}^{2}$ & $\mathrm{C}_{2} \mathrm{H} / \mathrm{HCN}$ & Silicate & PAH & $25 / 100 \mu \mathrm{m}$ \\
\hline $\mathrm{HNC} / \mathrm{HCN}$ & 1.00 & - & - & - & - & - \\
$\mathrm{HCO}^{+} / \mathrm{HCN}$ & -0.43 & 1.00 & - & - & - & - \\
$\mathrm{C}_{2} \mathrm{H} / \mathrm{HCN}$ & 0.46 & 0.09 & 1.00 & - & - & - \\
Silicate & -0.11 & -0.37 & 0.03 & 1.00 & - & - \\
$\mathrm{PAH}$ & -0.41 & 0.66 & 0.19 & -0.22 & 1.00 & - \\
$25 / 100 \mu \mathrm{m}$ & -0.24 & -0.10 & -0.2 & 0.03 & -0.46 & 1.00 \\
\hline
\end{tabular}

Table 4. Projection of the principal components onto the base of observables.

\begin{tabular}{lcccccc}
\hline \hline & PC 1 & PC 2 & PC 3 & PC 4 & PC 5 & PC 6 \\
\hline Variance \% & 45 & 29 & 16 & 6 & 3 & 1 \\
$\mathrm{HNC} / \mathrm{HCN}$ & 0.03 & -0.19 & 0.59 & -0.32 & 0.02 & -0.71 \\
$\mathrm{HCO}^{+} / \mathrm{HCN}$ & -0.24 & 0.17 & -0.26 & -0.18 & -0.9 & -0.21 \\
$\mathrm{C}_{2} \mathrm{H} / \mathrm{HCN}$ & -0.02 & 0.10 & 0.26 & -0.79 & -0.01 & 0.54 \\
Silicate & 0.83 & 0.52 & -0.06 & -0.06 & -0.07 & -0.12 \\
$\mathrm{PAH}$ & -0.46 & 0.68 & -0.20 & -0.17 & 0.42 & -0.28 \\
$25 / 100 \mu \mathrm{m}$ & 0.17 & -0.43 & -0.69 & -0.45 & 0.22 & -0.24 \\
\hline
\end{tabular}

Notes. For each PC, its contribution to the total variance in the data is also shown.

does not show any obvious correlation with galaxy properties and its interpretation is not straightforward. We will further discuss the results of the PC analysis in Paper II, when we will have additional information from VLA radio observations about, e.g., gas surface density and star-formation rate.

\subsection{Comparison with previous observations}

A previous study of the molecular emission of dense gas in luminous infrared galaxies was reported by Baan et al. (2008). The authors analyse data for 117 galaxies, with infrared luminosities ranging over about three orders of magnitude. Our diagrams in Fig. 4 can be directly compared with those of Fig. 9 in Baan et al. (2008).

The correlations between $\mathrm{HNC} / \mathrm{HCO}^{+}$and the two ratios $\mathrm{HNC} / \mathrm{HCN}$ and $\mathrm{HCO}^{+} / \mathrm{HCN}$, shown by Baan et al. (2008) in plots $a$ and $c$, are confirmed by our observations. Our measurement have a much smaller scatter, thanks to the excellent relative calibration of the line intensities provided by the EMIR receiver. 
However, we cannot exclude that the smaller scatter is partially caused by source selection effects.

Plot $b$ in Baan et al. (2008), showing $\mathrm{HCO}^{+} / \mathrm{HCN}$ versus $\mathrm{HNC} / \mathrm{HCN}$ cannot be reproduced by our data. In particular, we did not observe any galaxy with $\log \mathrm{HNC} / \mathrm{HCN}<-0.4$ and $\log$ $\mathrm{HCO}^{+} / \mathrm{HCN}<0$, while about $25 \%$ of the sources plotted by Baan et al. (2008) are in this range.

As a result, the correlation between $\mathrm{HCO}^{+} / \mathrm{HCN}$ and $\mathrm{HNC} / \mathrm{HCN}$ line ratios in our graph $b$ goes in the opposite direction, with low $\mathrm{HNC} / \mathrm{HCN}$ corresponding to high $\mathrm{HCO}^{+} / \mathrm{HCN}$. It is not clear whether this is caused by selection effects that may affect our observations, or to the large scatter in the data in Baan et al. (2008).

In Table C. 1 we compare line intensities from Baan et al. (2008) with our EMIR values. In most cases the two datasets agree at the $20 \%$ level, with some exceptions. The most striking differences are seen for $\mathrm{HNC} / \mathrm{HCN}$ in $\mathrm{NGC} 7469$ and $\mathrm{HCO}^{+} / \mathrm{HCN}$ in NGC 6240 , which vary more than $60 \%$, passing from values higher than unity in one dataset to much lower ratios in the other.

These discrepancies could be caused by calibration or pointing inaccuracies, which affect most narrow band single dish observations. The determination of line ratios with EMIR is more robust, because these inaccuracies do not affect the relative intensity of lines detected in the same band.

\section{Discussion}

\subsection{Interpreting line-intensity ratios}

Line-intensity ratios are often interpreted as estimates of the abundance ratios of two molecular species. This is true only if the emission is optically thin and the two molecules have similar excitation properties. Moreover, single dish observations only reveal global ratios, averaged on the whole galaxy, and the interpretation of the results has to take into account the lack of spacial information.

In our study we detect transitions with critical densities $\left(n_{\mathrm{c}}\right)$ that vary by almost four orders of magnitude (see Table 2 ), and it is therefore likely that excitation plays an important role in determining the observed ratios.

Transitions with large $n_{\mathrm{c}}$ are efficiently excited in high density gas, which is mostly concentrated in the inner parts of galaxies, while the emission of lines with lower $n_{\mathrm{c}}$, which can be excited in more diffuse gas, is generally more extended. Low- $n_{\mathrm{c}}$ lines will thus have a higher beam filling factor, resulting in an increased $T_{\mathrm{A}}^{\star}$. In this context, it is advisable to compare line intensities of transitions with similar $n_{\mathrm{c}}$, since the resulting ratios can be more directly related to molecular abundances.

Also, the excitation temperature of the transitions is an important factor when interpreting single dish intensities. If the emission is coming from regions with different gas temperatures, this translates into a different population of the low- $J$ levels, which directly affects the line intensity ratios.

Another complication arises when we take into account radiative excitation. Many of the observed molecules, as e.g. HNC, $\mathrm{HCN}$, and $\mathrm{HC}_{3} \mathrm{~N}$, can in fact be affected by radiative pumping via IR vibrational modes. Radiative pumping of $\mathrm{HNC}$ has been proposed by Aalto et al. (2007) as an explanation of the high $\mathrm{HNC} / \mathrm{HCN}$ ratio observed in LIRGs, while the strong connection of $\mathrm{HC}_{3} \mathrm{~N}$ excitation with the IR continuum has been discussed in Costagliola \& Aalto (2010).

Extreme caution should be used therefore when interpreting global line intensity ratios as abundance indicators. Molecular excitation across our galaxy sample will be further discussed in a following paper, where we will include $1 \mathrm{~mm}$ counterparts of the observed lines.

\subsection{Is $\mathrm{HCO}^{+} / \mathrm{HCN}$ driven by XDR chemistry?}

The $\mathrm{HCO}^{+} / \mathrm{HCN} J=1-0$ line ratio has been proposed as a reliable diagnostic tool to distinguish between AGN- and starburst-powered galaxies (e.g., Kohno et al. 2001; Imanishi et al. 2004, 2007). Interferometric observations by Kohno et al. (2001) reveal that Seyfert galaxies have lower $\mathrm{HCO}^{+} / \mathrm{HCN}$ ratios compared with starbursts. Seyfert galaxies with starburstlike $\mathrm{HCO}^{+} / \mathrm{HCN}$ ratios are generally interpreted as mixed AGNstarburst objects (Imanishi et al. 2007). In our observations, starbursts and LIRGs have, on average, higher $\mathrm{HCO}^{+} / \mathrm{HCN}$ ratios than AGN, which is consistent with the trend observed by Kohno et al. (2001) and Imanishi et al. (2007). This is commonly attributed to an enhancement of HCN abundance in the X-ray-dominated region (XDR, Maloney et al. 1996) surrounding an AGN (Lepp \& Dalgarno 1996). Chemical models by Meijerink \& Spaans (2005) show that an $\mathrm{HCO}^{+} / \mathrm{HCN}$ line ratio lower than one can be observed on the surface of low-density $\left(n<10^{5} \mathrm{~cm}^{-3}\right)$ XDRs, for $\mathrm{H}_{2}$ column densities $<10^{22} \mathrm{~cm}^{-2}$. However, at higher depths inside the molecular cloud, $\mathrm{HCO}^{+}$ abundance increases and eventually the cumulative $\mathrm{HCO}^{+} / \mathrm{HCN}$ column density ratio becomes higher than one. Meijerink et al. (2007) report that for $N\left(\mathrm{H}_{2}\right)>10^{23} \mathrm{~cm}^{-2}$, the intensity of the $J=1-0$ and $J=4-3$ transitions emerging from an XDR is higher for $\mathrm{HCO}^{+}$than for $\mathrm{HCN}$ by a factor of at least three. This is attributed to the fact that HCN abundance in an XDR is enhanced (compared with $\mathrm{HCO}^{+}$) only in a narrow range of ionization rate/density (Fig. 3 in Lepp \& Dalgarno 1996). Meijerink et al. (2007) conclude that a low $\mathrm{HCO}^{+} / \mathrm{HCN}$ line ratio is a good tracer of UV-dominated regions (PDR) for gas densities $>10^{5} \mathrm{~cm}^{-3}$, rather than X-ray-irradiated gas. Thus the observed low $\mathrm{HCO}^{+} / \mathrm{HCN}$ line ratio could be either caused by low-density $\left(n<10^{5} \mathrm{~cm}^{-3}\right)$ XDRs or dense $\left(n>10^{5} \mathrm{~cm}^{-3}\right)$ PDRs.

Observations by Blake et al. (1987) in the Orion molecular cloud reveal extremely low $\mathrm{HCO}^{+} / \mathrm{HCN}$ abundance ratios $\left(<10^{-3}\right)$ in hot cores, i.e. warm and dense gas around massive young stars. This is supported by calculations by Bayet et al. (2008), who find abundances of $\mathrm{HCO}^{+}$as low as $10^{-12}$ (relative to $\mathrm{H}_{2}$ ) in a wide range of hot core models. The faint $\mathrm{HCO}^{+}$emission may thus be caused by deeply embedded star-formation, instead of XDR or PDR chemistry.

This interpretation is based on the assumption that line ratios are directly linked to relative abundances. This may not be true for $\mathrm{HCN}$ and $\mathrm{HCO}^{+}$, which have different excitation properties. The critical density of the $J=1-0$ transition of $\mathrm{HCO}^{+}$ is one order of magnitude lower than for HCN. As a consequence, $\mathrm{HCO}^{+}$emission can originate from lower density gas, which in general has a higher filling factor compared with the dense $\left(n_{\mathrm{H}}>10^{5} \mathrm{~cm}^{-3}\right)$ component. This implies that the high $\mathrm{HCO}^{+} / \mathrm{HCN}$ line ratio observed in starburst galaxies may be caused by different filling factors for the two molecules instead of by their abundance.

Although Krips et al. (2008) suggest that the $\mathrm{HCN} / \mathrm{HCO}^{+}$ line ratio can often serve as a measure of abundance, we advise caution in doing so. Recent work by Sakamoto et al. (2009) and Aalto et al. (2009) show that in luminous, dusty nuclei absorption and radiative excitation affect the line emission of these molecules on global scales. 


\subsection{The $\mathrm{HNC/HCN}$ ratio}

In the Galaxy, the $\mathrm{HNC} / \mathrm{HCN}$ observed line ratio ranges from $1 / 100$ in hot cores (Schilke et al. 1992) to values as high as 4 in dark clouds (Hirota et al. 1998). The abundance of the HNC molecule decreases with increasing gas temperature. Hirota et al. (1998) suggest that this may be owing to the temperature dependence of neutral-neutral reactions, which, for temperatures exceeding $24 \mathrm{~K}$, selectively destroy $\mathrm{HNC}$ in favour of HCN. Interestingly, Greaves \& Nyman (1996) find HCN/HNC abundance ratios of up to 6 for Galactic spiral arm clouds (GMCs). The highest ratios are observed in regions of lowest $\mathrm{N}\left(\mathrm{H}_{2}\right)$, consistent with models by Schilke et al. (1992), which show and increase of $\mathrm{HCN} / \mathrm{HNC}$ with increasing $\mathrm{C} / \mathrm{CO}$. Bright HNC emission is commonly observed in extragalactic objects (Aalto et al. 2002; Wang et al. 2004; Meier \& Turner 2005; Pérez-Beaupuits et al. 2007). In particular, overluminous HNC $J=3-2$ is found in LIRGs (e.g., Aalto et al. 2007), where gas temperatures, derived by the IR dust continuum (Evans et al. 2003) and mid-IR molecular absorption (Lahuis et al. 2007), are usually $>50 \mathrm{~K}$ and can reach values as high as a few $100 \mathrm{~K}$. Here, ion-molecule chemistry in PDRs may be responsible for the observed ratios. Meijerink et al. (2007) find that the HNC/HCN $J=1-0$ line ratio is enhanced in PDRs and can reach a maximum value of one for $\mathrm{H}_{2}$ column densities exceeding $10^{22} \mathrm{~cm}^{-2}$. Even higher $\mathrm{HNC} / \mathrm{HCN}$ line ratios can result from XDR emission. Models by Meijerink et al. (2007) show that for the $J=4-3$ transition, this ratio can be as high as 1.6 in dense $\left(n(\mathrm{H})>10^{6} \mathrm{~cm}^{-3}\right) \mathrm{X}$-ray-dominated regions. The average $\mathrm{HNC} / \mathrm{HCN} J=1-0$ line ratio for AGN and LIRGs in our sample is $\simeq 0.5$ (see plot $c$ in Fig. 4), which is consistent with the results by Meijerink et al. (2007) for XDRs with densities $<10^{5} \mathrm{~cm}^{-3}$ and high X-ray fluxes, $F_{\mathrm{X}}>10 \mathrm{erg} \mathrm{cm}^{-2} \mathrm{~s}^{-1}$. For the same sources, low-density XDR chemistry could also explain the observed $\mathrm{HCO}^{+} / \mathrm{HCN}$ ratios. The $\mathrm{HNC} / \mathrm{HCN}$ ratio of order unity observed in UGC 5101 and NGC 2273 (see Table 1) is consistent with the emission coming from either a PDR or a low-density XDR $\left(n_{\mathrm{H}}<10^{5} \mathrm{~cm}^{-3}\right)$. Models of PDRs with different density and radiation field strength can explain $\mathrm{HNC} / \mathrm{HCN} J=1-0$ line ratios ranging from 0.5 to 1 (Meijerink \& Spaans 2005; Loenen et al. 2008). In our sample, the observed $\mathrm{HNC} / \mathrm{HCN}$ ratio reaches values as low as 0.2 (plots $b, c$ in Fig. 4), which cannot be explained by existing PDR or XDR models. However, line ratios $\mathrm{HNC} / \mathrm{HCN}<0.5$ are observed in Galactic PDRs. Abundance ratios of about 0.2 are found, e.g. , by Fuente et al. (1993) in the PDR region of the reflection nebula NGC 7023. Low values of $\mathrm{HNC} / \mathrm{HCN}$ are also found in other galaxies (see, e.g., Baan et al. 2008) as discussed in Sect. 4.4.

\subsection{Luminous $\mathrm{HC}_{3} \mathrm{~N}$ : a nascent starburst tracer?}

Bright $\mathrm{HC}_{3} \mathrm{~N}$ emission is often observed in Galactic hot cores (Rodriguez-Franco et al. 1998; de Vicente et al. 2000). The molecule is easily destroyed by UV radiation and reactions with $\mathrm{C}^{+}$and $\mathrm{He}^{+}$(e.g., Turner et al. 1998) and can only survive in regions shielded by large gas and dust columns. In a highresolution study of the chemistry of IC 342, Meier \& Turner (2005) find that $\mathrm{HC}_{3} \mathrm{~N}$ emission follows the $3 \mathrm{~mm}$ continuum dust emission and anti-correlates with regions of intense UV radiation, such as PDRs. Observations in the Galaxy (Wyrowski et al. 1999) and in the LIRG NGC 4418 (Aalto et al. 2007; Costagliola \& Aalto 2010) show that $\mathrm{HC}_{3} \mathrm{~N}$ is strongly connected with the IR field by means of its vibrational bending modes. In our sample, only five galaxies have detectable $\mathrm{HC}_{3} \mathrm{~N}$ emission: NGC 4418, IRAS 17208, IC 860, NGC 1068, and NGC 7771. For completeness, we also include in our analysis the detection of $\mathrm{HC}_{3} \mathrm{~N} J=10-9$ in the ULIRG Arp 220 by Aalto et al. (2002). Since these objects span a wide range of LSR velocities, in most cases the detectability of $\mathrm{HC}_{3} \mathrm{~N}$ does not depend on the sensitivity of our measurements, but on intrinsic properties of the observed galaxies. This is further discussed in Appendix A, where we compare $\mathrm{HCN}$ and $\mathrm{HC}_{3} \mathrm{~N}$ detection thresholds.

All galaxies with detected $\mathrm{HC}_{3} \mathrm{~N}$ have $\mathrm{HCO}^{+} / \mathrm{HCN}<1$ and higher-than-average $\mathrm{HNC} / \mathrm{HCN}$ ratios. This is consistent with the hot core models described in Bayet et al. (2008), as discussed in Sect. 5.2. It is thus possible that the bulk of the molecular emission in these objects comes from regions of young starformation. This interpretation was already proposed by Aalto et al. (2007) to explain the $\mathrm{HC}_{3} \mathrm{~N}$ emission of $\mathrm{NGC} 4418$ in terms of a nascent starburst.

The $\mathrm{HC}_{3} \mathrm{~N}$-bright galaxies reside in the $\mathrm{HCO}^{+} / \mathrm{HCN}$ interval that in the diagnostic diagrams discussed by Kohno et al. (2001) is associated with Seyfert galaxies. Kohno et al. (2001) and Imanishi et al. (2007) interpret the observed ratios as the signature of XDR chemistry driven by the hard radiation from the AGN. Our data seem to challenge this interpretation, since $\mathrm{HC}_{3} \mathrm{~N}$ cannot survive in significant abundance in highly irradiated gas. Bright $\mathrm{HC}_{3} \mathrm{~N}$ emission can emerge from an AGN only if the central source is shielded by a considerable amount of gas and dust. In these objects, the bulk of the molecular emission would be coming from an hot core-like region instead of being dominated by XDR chemistry. Plots $e$ and $f$ in Fig. 4 also show that the galaxies with the highest $\mathrm{HC}_{3} \mathrm{~N} / \mathrm{HCN}$ ratios have high ratios of IRAS 60 to $100 \mu \mathrm{m}$ fluxes. This may indicate that $\mathrm{HC}_{3} \mathrm{~N}$ is more abundant in warm environments.

An enhanced $\mathrm{HC}_{3} \mathrm{~N}$ emission at high gas temperatures could also be owing to excitation effects. The upper state energy of the observed $\mathrm{HC}_{3} \mathrm{~N}$ transitions is indeed of the order of 20-30 K, higher by a factor of $\simeq 5$ than typical energies for other high density tracers, meaning that the molecule is more efficiently excited at higher temperatures. The bright $\mathrm{HC}_{3} \mathrm{~N}$ emission is thus likely to be a combination of enhanced abundance and favourable excitation conditions in warm, dense gas.

Radiative pumping of the molecule's rotational levels by means of IR vibrational modes was also suggested by Costagliola \& Aalto (2010).

\section{5. $\mathrm{CO} /{ }^{13} \mathrm{CO}$ vs. dust temperature}

Plot $g$ in Fig. 5 shows how the ratio $\mathfrak{R}_{1-0} \equiv \mathrm{CO} /{ }^{13} \mathrm{CO} J=1-0$ varies with dust temperature. The dust temperature was calculated from IRAS fluxes at 100 and $60 \mu \mathrm{m}$, via the formula

$T_{\text {dust }}=-(1+z)\left[\frac{82}{\ln \left(0.3 f_{60 \mu \mathrm{m}} / f_{100 \mu \mathrm{m}}\right)}-0.5\right]$,

see, e.g., Solomon et al. (1997). The IRAS fluxes were obtained from the NASA/IPAC Extragalactic Database.

A clear trend is observed, with $\mathfrak{R}_{1-0}$ increasing as $T_{\text {dust }}$ increases. This is a well established result, see, e.g., Young \& Sanders (1986) and Aalto et al. (1995) for a detailed discussion. The trend is generally attributed to opacity effects arising in a collection of non-uniform clouds, averaged in the beam. In a molecular cloud, the emission of $\mathrm{CO} 1-0$ arises mostly from the outer layers, up to optical depths $\tau \simeq 1$, while the ${ }^{13} \mathrm{CO}$ emission stays in general optically thin deeper into the volume of the 
cloud. The line ratio $\mathfrak{R}_{1-0}$ is thus mostly affected by the relative opacity of the two species.

The opacity of the CO 1-0 line strongly depends on the excitation temperature (see, e.g., Aalto et al. 1995, Eq. (1)). With rising $T_{\text {ex }}$, the $J=1$ level is in fact de-populated, causing a decrease in optical depth of the CO 1-0 transition. Where $\tau_{\mathrm{CO}} \simeq 1$, we can assume $\tau_{13} \mathrm{CO} \ll 1$ and the intensity of the ${ }^{13} \mathrm{CO} J=1-$ 0 line is an even steeper function of $T_{\text {ex }}$ than the ${ }^{12} \mathrm{CO}$ intensity (see, e.g., Mauersberger \& Henkel 1993). As a consequence, $\mathfrak{R}_{1-0}$ must increase with temperature.

In our Galaxy Young \& Sanders (1986) find that $\mathfrak{R}_{1-0}$ has an average value of roughly 8 , while, in extragalactic surveys, values $\mathfrak{R}_{1-0}>20$ are observed (e.g., Aalto et al. 1995; this work), the highest ratios being found in interacting systems and mergers. In our sample, the highest $\mathfrak{R}_{1-0} \simeq 28$ is found for the galaxies NGC 6240 and NGC 1614. In these objects, high gas temperatures and a diffuse ISM may result in an extremely low optical depth of the CO 1-0 line, which would explain the observed ratios.

Additional processes have been proposed in order to explain these extreme ratios. In the central regions of merging and interacting galaxies, large amounts of gas and dust are trapped in the deep potential well, giving rise to high gas pressures. Large turbulent motions, powered by differential rotation or stellar winds from an enhanced star-formation are required to sustain the clouds against collapse. This would cause a broadening of the $\mathrm{CO}$ emission line and a decrease in optical depth, with a consequent increase in $\mathfrak{R}_{1-0}$ (Aalto et al. 1995).

Chemistry could also provide an explanation of the observed high ratios. In star-forming regions the surfaces of molecular clouds are indeed dominated by UV-photons from young stars. In these regions, ${ }^{13} \mathrm{CO}$ is dissociated more easily than $\mathrm{CO}$, which is less affected by the UV radiation thanks to self-shielding (Aalto et al. 1995). This process could be relevant in starburst galaxies, as NGC 6240 and NGC 1614 in our sample.

The age of the starburst could also have an influence on $\mathfrak{R}_{1-0}$. Nuclear processing in stars favours ${ }^{13} \mathrm{CO}$ over $\mathrm{CO}$, and the evidence for an enhanced $\mathfrak{R}_{1-0}$ at low metallicities has been reported by, e.g., Young \& Sanders (1986). A large $\mathfrak{R}_{1-0}$ would be thus compatible with a young star-formation age.

\section{6. $\mathrm{CO} / \mathrm{CN}$ vs. $\mathrm{CO} / \mathrm{C}^{18} \mathrm{O}$}

Plot $h$ in Fig. 5 shows the ratio $\mathrm{CO} / \mathrm{CN}$ vs. $\mathrm{CO} / \mathrm{C}^{18} \mathrm{O}$. The total $\mathrm{CN}$ intensity was obtained by adding the integrated intensities of the two lines of the spin doublet $J=3 / 2-1 / 2$ and $J=1 / 2-1 / 2$. The ratio $\mathrm{CO} / \mathrm{CN}$ varies in our sample over roughly one order of magnitude and shows a general trend of increasing $\mathrm{CO} / \mathrm{CN}$ with increasing $\mathrm{CO} / \mathrm{C}^{18} \mathrm{O}$. This implies that $\mathrm{CN}$ and $\mathrm{C}^{18} \mathrm{O}$ emission are positively correlated. There are several potential explanations of this:

- we can assume that both $\mathrm{C}^{18} \mathrm{O}$ and $\mathrm{CN}$ are optically thin, for $\mathrm{CN}$ we know this from the $\mathrm{CN} 1-0$ spin group ratio, which is at the optically thin level for all detected galaxies (apart from, possibly, UGC 5101). In this case, the intensity of CN and $\mathrm{C}^{18} \mathrm{O}$ would scale with the total molecular column density. This linear scaling with total column would not happen for the CO 1-0 intensity, which can be considered to be at least moderately optically thick in all the galaxies in our sample. The observed correlation would then be a result of optical depth effects;

- it is also possible to speculate on other explanations. It is not immediately self-evident that the $\mathrm{C}^{18} \mathrm{O}$ and $\mathrm{CN}$ column densities should follow each other. In PDRs, the $\mathrm{CN}$ molecule is usually abundant up to a visual extinction $A_{\mathrm{V}}=2$. At higher depths in the clouds the radical CN may react with other molecules to form - for example - HCN. Thus, CN can, to some degree, trace the amount of PDR surfaces.

In PDRs, it is likely that $\mathrm{C}^{18} \mathrm{O}$ could become photodissociated. However, if the $\mathrm{CN}$ is tracing PDRs, it is also tracing the impact of the star-formation on host clouds. These are the regions where the ISM enrichment caused by the starburst hits first., i.e., where $\mathrm{C}^{18} \mathrm{O}$ would become enhanced early on. Hence, in this scenario, a $\mathrm{C}^{18} \mathrm{O}-\mathrm{CN}$ correlation would be expected as a result of ISM enrichment from the starburst.

In this context, it is interesting to note the distinct deviations from this correlation. We have the three outliers with faint $\mathrm{C}^{18} \mathrm{O}$ compared with their CN luminosities: NGC 4194, NGC 1614 and NGC 7469. A possible way to obtain this comparable overluminosity in $\mathrm{CN}$ is to have $\mathrm{CN}$ emerging from an AGN component, with no associated enrichment from a starburst component.

The $\mathrm{C}^{18} \mathrm{O}$ enrichment should come quickly in a starburst. If ${ }^{18} \mathrm{O}$ is a product of $\alpha$-capture on ${ }^{14} \mathrm{~N}$ during He burning, it will come from mass loss in He-burning systems, such as Wolf-Rayet stars and SNe type II. This means that it should come up with prompt nucleosynthesis - e.g., follow $\mathrm{O}$ abundance.

Because the molecular emission from NGC 7469 is dominated by the starburst ring, this comparatively $\mathrm{C}^{18} \mathrm{O}$ weakness is somewhat puzzling and should be further investigated. The observed high $\mathrm{CO} / \mathrm{C}^{18} \mathrm{O}$ ratio could also be caused by a low optical depth of the CO 1-0 line.

The other two outliers are different since they are both mergers where the $\mathrm{CO}$ emission is dominated by a prominent crossing dust lane and not the starburst region (Olsson et al. 2010; Aalto \& Hüttemeister 2000). It is therefore possible that the molecular gas in the IRAM beam is dominated by in-falling gas in the dust lane, which has not yet been enriched by the starburst. Indeed, Martín et al. (2010) find high $\mathrm{C} / \mathrm{C}^{13}$ ratios from $\mathrm{CO}$ and $\mathrm{CCH}$ observations in the starbursts M 82 and NGC 253, suggesting that the bulk of the molecular mass in these galaxies mostly consists of unprocessed material.

An outrigger in a different direction is IC 860. This galaxy has the lowest $\mathrm{CO} / \mathrm{C}^{18} \mathrm{O}$ line ratio in the sample - but the highest $\mathrm{CO} / \mathrm{CN} 1-0$ line ratio. In this source, it is possible that only a small fraction of the cloud surface area is in the form of PDRs and/or that the $\mathrm{CN}$ is being used up in forming $\mathrm{HC}_{3} \mathrm{~N}$ together with $\mathrm{C}_{2} \mathrm{H}_{2}$, which is boiling off the grains. Its luminous PAH emission (see Fig. 4d) does suggest however that PDRs should be quite abundant. It is possible that the PDRs in this object do not coexist with regions dense enough to excite $\mathrm{CN}$ emission.

\subsection{Other isotopic line ratios}

For a small subset of galaxies, it was possible to obtain isotopic line ratios for $\mathrm{HCN}$ and $\mathrm{HCO}^{+}$, which are reported in Table 5. If we assume a Galactic $\mathrm{C} /{ }^{13} \mathrm{C}$ abundance ratio of $\simeq 50$ (see, e.g., Wilson \& Rood 1994), or as high as 100 (Martín et al. 2010), it is evident that in the galaxies where ${ }^{13} \mathrm{C}$ variants were detected, $\mathrm{HCN}$ and $\mathrm{HCO}^{+} J=1-0$ transitions are at least moderately optically thick, with $\mathrm{HC} \star / \mathrm{H}^{13} \mathrm{C} \star$ as low as 4 . These data show how the assumption of optically thin lines when interpreting extragalactic molecular intensity ratios is not always correct and opacity effects have to be included in the analysis, even for species much less abundant than $\mathrm{CO}$. The broad band 
Table 5. Isotopic line intensity ratios for $\mathrm{HCN}$ and $\mathrm{NCO}^{+}$.

\begin{tabular}{lcc}
\hline \hline Galaxy & $\mathrm{HCN} / \mathrm{H}^{13} \mathrm{CN}$ & $\mathrm{HCO}^{+} / \mathrm{H}^{13} \mathrm{CO}^{+}$ \\
\hline NGC 1614 & $>2.8$ & $4(2)$ \\
Mrk 231 & $6(2)$ & $7(6)$ \\
NGC 4418 & $8(3)$ & $>4$ \\
NGC 1068 & $17(7)$ & $24(20)$ \\
\hline
\end{tabular}

Notes. Uncertainties $(1-\sigma)$ are shown in parenthesis.

Table 6. Detected lines of $\mathrm{HC}_{3} \mathrm{~N}$ in IC 860 .

\begin{tabular}{lcccc}
\hline \hline Transition & $\begin{array}{c}v \\
{[\mathrm{GHz}]}\end{array}$ & $\begin{array}{c}\int T_{\mathrm{A}}^{\star} \mathrm{d} v \\
{\left[\mathrm{~K} \mathrm{~km} \mathrm{~s}^{-1}\right]}\end{array}$ & $\begin{array}{c}\Delta v \\
{\left[\mathrm{~km} \mathrm{~s}^{-1}\right]}\end{array}$ & $\begin{array}{c}E_{\mathrm{up}} \\
{[\mathrm{K}]}\end{array}$ \\
\hline$J=10-9$ & 90.978 & $0.43(0.09)$ & 200 & 24 \\
$J=10-9 v_{7}=1 f$ & 91.333 & $<0.12$ & 170 & 342 \\
$J=12-11$ & 109.173 & $0.75(0.16)$ & 300 & 34 \\
$J=12-11 v_{7}=1 e$ & 109.441 & $0.33(0.08)$ & 170 & 355 \\
$J=12-11 v_{7}=1 f$ & 109.598 & $0.21(0.08)$ & 170 & 355 \\
\hline
\end{tabular}

Notes. The integrated intensity is not corrected for main beam efficiency (see Table 2).

of EMIR allows us to determine isotopic intensity ratios with unprecedented accuracy, which will be of great importance in constraining models of galactic emission. However, deeper integrations are still needed to set reliable limits in more optically thin environments. We also advise caution when interpreting $\mathrm{HCO}^{+} / \mathrm{H}^{13} \mathrm{CO}^{+}$intensity ratios, since the $\mathrm{H}^{13} \mathrm{CO}^{+} J=1-0$ line is blended with $\mathrm{SiO}$ emission and the two lines are not easy to separate at low SNRs.

\section{8. $\mathrm{HC}_{3} \mathrm{~N}$ in IC 860}

In total we detected four emission lines of $\mathrm{HC}_{3} \mathrm{~N}$ in IC 860, reported in Table 6 . In the $112 \mathrm{GHz}$ band, we detected two transitions of the vibrationally excited state $v_{7}=1(e, f)$, together with the $J=12-11, v=0$ line. In the $88 \mathrm{GHz}$ band we detected the $J=10-9, v=0$ line, and we have a tentative detection of the $v_{7}=1 f$, vibrationally excited transition.

The interaction between the bending angular momentum of the vibrationally excited states and the rotational angular momentum of the molecule leads to a 1-splitting of the levels. Each vibrational state is thus split into two levels, labelled $e$ or $f$ depending on the wavefunctions parity properties. These parity labels are also shown in Table 6. A useful reference for the labelling of doubled levels in linear molecules is Brown et al. (1975).

Both $e$ and $f$ states have similar excitation properties and should result in an emission doublet with comparable line intensities. In our spectra, this is true for the $J=12-11 v_{7}=1 e$ and $v_{7}=1 f$ lines at $109 \mathrm{GHz}$, which show integrated line intensities within the measurement errors. The $J=10-9 v_{7}=1 f$ transition at $91 \mathrm{GHz}$ has no detected $e$ counterpart and will therefore be treated as an upper limit in our excitation analysis.

A first analysis of the excitation of the molecule was performed via the population diagram method. This results in an excitation temperature of $15 \mathrm{~K}$ for the $v=0$ transitions and $42 \mathrm{~K}$ for the $v_{7}=1$ lines.

A rotational temperature of $15 \mathrm{~K}$ is significantly lower than the $30 \mathrm{~K}$ derived by Costagliola \& Aalto (2010) for the other $\mathrm{HC}_{3} \mathrm{~N}$-bright LIRG, NGC 4418. This may suggest that bright
$\mathrm{HC}_{3} \mathrm{~N}$ emission could originate from different environments. However, our estimate is based only on two transitions and has to be further investigated.

The vibrational excitation of $\mathrm{HC}_{3} \mathrm{~N}$ is strongly dependent on the IR field, since the molecule's bending modes can only be excited by radiation. A higher excitation of the $v_{7}=1$ lines, compared with the $v=0$ levels, is therefore expected in a source with a strong IR emission such as IC 860 . The derived $42 \mathrm{~K}$ is a lower limit to the excitation temperature, because we only have an upper limit for the $J=10-9 v_{7}=1 f$ transition at $91 \mathrm{GHz}$.

In highly obscured galaxies, the detected IR emission is in most of the cases optically thick. Because millimeter radiation can penetrate a larger column of gas and dust, vibrationally excited $\mathrm{HC}_{3} \mathrm{~N}$ is a valuable probe of the IR field beyond its optically thick surface.

The first multi-transition analysis of extragalactic vibrationally excited $\mathrm{HC}_{3} \mathrm{~N}$ was reported by Costagliola \& Aalto (2010) for the IR galaxy NGC 4418. Here the authors find a $\mathrm{HC}_{3} \mathrm{~N}$ vibrational temperature of $500 \mathrm{~K}$, much higher than the temperature of the optically thick dust (80 K, Evans et al. 2003), which may be the evidence of an obscured compact object in the centre of the galaxy.

It would be interesting to perform this kind of analysis on IC 860, which presents unresolved emission in both IR and radio and would then be a good candidate for a deeply embedded compact source.

\subsection{Models of galactic molecular emission}

The line ratios observed by Baan et al. (2008) were compared with a grid of XDR and PDR models by Loenen et al. (2008). These authors find that the low $\mathrm{HNC} / \mathrm{HCN}<0.5$ line ratios cannot be explained by XDR or PDR chemistry, and they claim that a possible explanation could come from mechanical heating. At temperatures $>30 \mathrm{~K}$, neutral-neutral reactions efficiently destroy HNC in favour of HCN (Hirota et al. 1998). Loenen et al. (2008) suggest that shocks from supernova explosions may heat the gas to temperatures $>100 \mathrm{~K}$, resulting in a depletion of HNC. The observed $\mathrm{HNC} / \mathrm{HCN}$ line ratios can be explained by mechanical heating for a gas density of $10^{4.5}$ and a star-formation rate (SFR) of $20 M_{\odot} \mathrm{yr}^{-1}$. Higher densities would require higher SFR, which would result in the destruction of the molecular material surrounding the star-formation region (Loenen et al. 2008). In this scenario, the observed low $\mathrm{HNC} / \mathrm{HCN}$ line ratio would originate from low-density $\left(n_{\mathrm{H}}<10^{5} \mathrm{~cm}^{-3}\right)$, warm ( $T \simeq 100 \mathrm{~K})$ gas. The corresponding high $\mathrm{HCO}^{+} / \mathrm{HCN}$ observed in plot Fig. $4 b$ may be a consequence of the low gas density. As discussed in Sect. 5.2, $\mathrm{HCO}^{+} 1-0$ has a lower critical density $\left(\simeq 10^{4} \mathrm{~cm}^{-3}\right)$ compared with $\mathrm{HCN} 1-0\left(\simeq 10^{5} \mathrm{~cm}^{-3}\right)$ and is more efficiently excited in diffuse gas. An increased $\mathrm{HCO}^{+}$abundance may also be caused by dissipation of turbulence driven by the supernovae winds. Models by Joulain et al. (1998) show that in low density gas, bursts of viscous dissipation can heat the gas up to the high temperatures required by endothermic reactions, such as $\mathrm{C}^{+}+\mathrm{H}_{2} \rightarrow \mathrm{CH}^{+}+\mathrm{H}(\Delta E / k \simeq 4600 \mathrm{~K})$, more efficient than the standard cosmic ray-driven chemistry. This leads to an enhancement of $\mathrm{CH}^{+}, \mathrm{OH}$, and $\mathrm{HCO}^{+}$, and may help to create the high $\mathrm{HCO}^{+} / \mathrm{HCN}$ ratios we observe.

This picture is complicated by models of $\mathrm{HCN}$ and $\mathrm{HNC}$ chemistry as discussed in Schilke et al. (1992). For gas temperatures $\simeq 100 \mathrm{~K}$, an efficient destruction of HNC by neutral-neutral reactions requires densities higher than $10^{5} \mathrm{~cm}^{-3}$, while at lower densities the HCN/HNC abundance ratio is about unity (Fig. 13 in Schilke et al. 1992). However, at higher gas temperatures 
( $>200 \mathrm{~K})$, HNC destruction may become effective even for densities as low as $\simeq 10^{4} \mathrm{~cm}^{-3}$ and thus provide an explanation for the low $\mathrm{HNC} / \mathrm{HCN}$.

\subsection{0. $\mathrm{HCO}^{+}, \mathrm{HNC}$, and PAHs}

In Fig. $4 d$, we compare the observed $\mathrm{HNC} / \mathrm{HCO}^{+} J=1-0$ line ratios with the $\mathrm{PAH}$ equivalent widths $(\mathrm{EW})$ from (Spoon et al. 2007). The distribution of $\mathrm{HNC} / \mathrm{HCO}^{+}$can be described as a step function: it is roughly constant for low PAH EWs and has a sudden drop for $\log _{10} E W>-0.5$. The galaxies with high PAH EWs are mostly starbursts or LIRGS.

PAHs are excited by UV radiation and destroyed by hard radiation (e.g., X-rays). Because of this, strong PAH emission is usually interpreted as a clear signature of star-formation, instead of AGN activity. A decrement of $\mathrm{HNC} / \mathrm{HCO}^{+}$for high PAH EWs may then imply either that $\mathrm{HCO}^{+}$is enhanced in a PDR, or that $\mathrm{HNC}$ is depleted. Indeed, if we compare the $\mathrm{HCO}^{+}$and $\mathrm{HNC}$ emission to $\mathrm{HCN} 1-0$, for increasing $\mathrm{PAH}$ EWs we have an increase in the $\mathrm{HCO}^{+} / \mathrm{HCN}$ ratio and a decrease in $\mathrm{HNC} / \mathrm{HCN}$. This can be seen in the graphs in Fig. B.1.

If directly connected to the molecular abundances, the observed ratios are difficult to explain with PDR and XDR chemistry. Models by Meijerink \& Spaans (2005) show that $\mathrm{HCO}^{+} / \mathrm{HCN}$ is lower in PDRs than in XDRs, and $\mathrm{HNC} / \mathrm{HCN}$ very close to unity in a wide range of densities and gas temperatures. However, the observed trend agrees with results by Imanishi et al. (2007), who find that AGNs have a lower $\mathrm{HCO}^{+} / \mathrm{HCN}$ ratio than starburst galaxies.

A depletion of HNC is also expected in shocks. Here, because of the high temperatures and densities, neutral-neutral reactions efficiently destroy $\mathrm{HNC}$ in favour of $\mathrm{HCN}$ (Schilke et al. 1992). Mechanical heating from shocks generated by supernova explosions may explain the observed trend. In this case, the $\mathrm{HNC} / \mathrm{HCN}$ ratio should follow the dependence on temperature of neutral-neutral reactions. In Fig. $4 e$ we compare the $\mathrm{HNC} / \mathrm{HCN}$ ratio with the ratio of the IRAS fluxes at 60 and $100 \mu \mathrm{m}$. The graph clearly shows that there is no strong dependence of the $\mathrm{HNC} / \mathrm{HCN}$ ratio with dust temperature. At densities $>10^{5} \mathrm{~cm}^{-3}$, gas and dust thermalise and this should show in the diagram, since neutral-neutral reactions strongly depend on gas temperature. At densities below $10^{5} \mathrm{~cm}^{-3}$, the thermal coupling becomes poor, however, and it is possible to have $T_{\text {gas }}>$ $T_{\text {dust }}$. This scenario is complicated by observations of $\mathrm{HCO}^{+}$in shocked gas in the Galaxy (Jørgensen et al. 2004), which reveal that the molecule is destroyed by reactions with $\mathrm{H}_{2} \mathrm{O}$ in the shock front. Detectable $\mathrm{HCO}^{+}$abundances are found in the aftermath of the shock, where water freezes on grains. Jørgensen et al. (2004) report an $\mathrm{HCO}^{+} / \mathrm{HCN}$ column density ratio of 0.6 in the outflow of the low-mass protostar NGC 1333 IRAS 2A.

\section{Conclusions}

We observed the molecular emission of 23 galaxies with the new EMIR receiver at IRAM $30 \mathrm{~m}$ in the frequency bands $88 \mathrm{GHz}$ and $112 \mathrm{GHz}$. The observed line ratios were compared with existing models of emission from PDRs, XDRs, and hot cores. All non-compact starburst galaxies are found to be $\mathrm{HCO}^{+}$-luminous, with line ratios $\mathrm{HCO}^{+} / \mathrm{HCN} \geq 1$. Luminous infrared galaxies and Seyferts do not show any strong trend. However, the majority of active galactic nuclei do reside at low $\mathrm{HCO}^{+} / \mathrm{HCN}$ values compared with starbursts.

When $\mathrm{HNC} / \mathrm{HCO}^{+}$increases, galaxies move towards higher $\mathrm{HNC} / \mathrm{HCN}$ and $\mathrm{HCO}^{+} / \mathrm{HCN}$ ratios. Galaxies with
$\mathrm{HCO}^{+} / \mathrm{HCN}<1$ present on average a $30 \%$ increase in $\mathrm{HNC} / \mathrm{HCN}$, compared with those at higher $\mathrm{HCO}^{+} / \mathrm{HCN}$ ratios. If we exclude the Seyfert NGC 2273, the brightest HNC emission is found in luminous infrared galaxies. Starbursts are generally characterized by faint $\mathrm{HNC}$ and bright $\mathrm{HCO}^{+}$emission (compared with $\mathrm{HCN}$ ), while LIRGs mostly occupy the opposite end of the $\mathrm{HNC}-\mathrm{HCO}^{+}$correlation, with high $\mathrm{HNC}$ and low $\mathrm{HCO}^{+}$intensities. All HNC-bright galaxies are either LIRGs or Seyferts.

All $\mathrm{HC}_{3} \mathrm{~N}$ detections have $\mathrm{HCO}^{+} / \mathrm{HCN}<1$ and $\mathrm{HNC} / \mathrm{HCN}>0.4$. Most of these are are LIRGs, the only exception being the Seyfert NGC 1068.

Starburst galaxies tend to have high $\mathrm{HCO}^{+} / \mathrm{HCN}$ and high PAH EWs, in agreement with observations by Kohno et al. (2001) and Imanishi et al. (2007).

The interpretation of the low $\mathrm{HCO}^{+} / \mathrm{HCN}$ ratio as an effect of XDR chemistry (Imanishi et al. 2007) is inconsistent with our observations, since we find bright $\mathrm{HC}_{3} \mathrm{~N}$ emission in galaxies with the lowest $\mathrm{HCO}^{+} / \mathrm{HCN}$. The $\mathrm{HC}_{3} \mathrm{~N}$ molecule is easily destroyed by reactions with ions and hard UV and is thus extremely unlikely to survive in X-ray dominated environments.

Models by Bayet et al. (2008) for hot cores provide a better explanation for the observed ratios, suggesting that bright $\mathrm{HC}_{3} \mathrm{~N}$ and faint $\mathrm{HCO}^{+}$emission might be good tracers of embedded star-formation.

Vibrationally excited $\mathrm{HC}_{3} \mathrm{~N}$ was detected in IC 860, where it was possible to obtain an estimate of the rotational temperature of the $v=0$ transitions of $15 \mathrm{~K}$, and an upper limit for the $v_{7}=1$ levels of $42 \mathrm{~K}$. This galaxy has the highest $\mathrm{HC}_{3} \mathrm{~N} / \mathrm{HCN}$ ratio of the sample, representing an interesting target for further investigation of the molecule's properties.

In agreement with previous studies, we find that the $\mathrm{CO} /{ }^{13} \mathrm{CO}$ intensity ratio is positively correlated with dust temperature, with typical values $\simeq 20$, higher than the ones observed in the Galaxy. We suggest that these high values can be caused by excitation (high temperatures, diffuse gas) and/or abundance effects.

The emission of $\mathrm{CN}$ and $\mathrm{C}^{18} \mathrm{O}$ appear to be positively correlated. This may simply be because both $\mathrm{CN}$ and $\mathrm{C}^{18} \mathrm{O}$ are optically thin and scale with the total gas column, or may be caused by ISM enrichment by the starburst activity. $\mathrm{CN}$ emission is, indeed, particularly strong in PDR surfaces, which are supposed to be tracing the impact of star-formation on the host clouds.

In agreement with previous extragalactic studies (e.g., Baan et al. 2008), we find $\mathrm{HNC} / \mathrm{HCN}$ intensity ratios lower than 0.4 , which cannot be explained by current models of PDR and XDR chemistry. A possible explanation was suggested by Loenen et al. (2008) to be mechanical heating by supernova explosions. This model would require low gas densities $\left(<10^{5} \mathrm{~cm}^{-3}\right)$ or extremely high star-formation rates $\left(>20 M_{\odot} \mathrm{yr}^{-1}\right)$, but would explain both the low $\mathrm{HNC} / \mathrm{HCN}$ and high $\mathrm{HCO}^{+} / \mathrm{HCN}$ ratios in starbursts. Mechanical heating by SN explosions could also explain the observed drop of the $\mathrm{HNC} / \mathrm{HCO}^{+}$ratio for $\mathrm{PAH}$ EWs $>$ $0.3 \mu \mathrm{m}$, if we assume that PAHs efficiently trace star-formation.

However, a temperature dependence of the $\mathrm{HNC} / \mathrm{HCN}$ ratio, which would be expected if the HNC abundance was depleted by neutral-neutral reaction in the shocked gas, does not emerge from our data. Furthermore, it is not yet clear how SN shocks affect $\mathrm{HCO}^{+}$abundance, with different models giving contrasting results. 


\subsection{Future directions}

Evidently, explaining the molecular emission observed in galaxies by means of the existing chemical models is a challenging task. Our understanding of the gas physical conditions is still affected by many uncertainties. Mechanical heating, invoked to explain the low $\mathrm{HNC} / \mathrm{HCN}$ ratios, requires further investigation with shock models and observations of shock tracers (e.g., IR transitions of $\mathrm{H}_{2}$ or $\mathrm{SiO}$ ). For single dish observations, the analysis of the observed emission is complicated by the lack of spatial information. Radiation from different molecules and transitions may in fact be coming from different regions in the galaxy, and thus it may be impossible to explain observations with only one set of physical conditions. Spatial distribution and density gradients in the molecular gas may have a strong impact on the observed line ratios. This may be particularly important for the $\mathrm{HCO}^{+} / \mathrm{HCN}$ ratio, which appears to be strongly densitydependent, because of both chemical and excitation effects. The angular resolution needed to resolve the spatial distribution of molecular gas in distant galaxies will become available in the foreseeable future with the ALMA interferometer. Observations of mm lines of formaldehyde $\left(\mathrm{H}_{2} \mathrm{CO}\right)$ may also help to constrain density and temperature in these objects. Sub-mm observations of high- $J$ transitions of $\mathrm{CO}$, which have been indicated as excellent tracers of PDRs by Meijerink et al. (2007), are now available with the Herschel telescope. The increased sensitivity of new generation $\mathrm{mm}$ - and sub-mm observatories will allow us to detect less abundant molecular species (e.g., $\mathrm{HNCO}, \mathrm{CH}_{3} \mathrm{OH}$ ) that may be better tracers of interstellar chemistry (e.g., Meier \& Turner 2005; Martín et al. 2009). The evolution of the starburst phase can be studied with radio continuum observations, which will be discussed in Paper II. We also plan to further explore molecular excitation in our sample by means of multitransition studies. We successfully applied for telescope time at IRAM $30 \mathrm{~m}$ to observe $1 \mathrm{~mm}$ lines of the species discussed in the present work. Collected data will be discussed in Paper III, where we will present a NLTE analysis of the emission of the main molecular species, together with a comparison with a grid of PDR and XDR models.

Acknowledgements. We thank the staff at the IRAM $30 \mathrm{~m}$ telescope for their kind help and support during our observations. Furthermore, we would like to thank the IRAM PC for their generous allocation of time for this project.

This research was supported by the EU Framework 6 Marie Curie Early Stage Training programme under contract number MEST-CT-2005-19669 "ESTRELA" and by the European Community Framework Programme 7, Advanced Radio Astronomy in Europe, grant agreement no. 227290, "RadioNet". A.A., M.A.P.T. and M.R. acknowledge support by the Spanish MICINN through grant AYA2009-13036-CO2-01. P.v.d.W. would like to thank the Scottish Universities Physics Alliance (SUPA) for support through a Distinguished Visitor Grant.

\section{References}

Aalto, S., \& Hüttemeister, S. 2000, A\&A, 362, 42

Aalto, S., Booth, R. S., Black, J. H., \& Johansson, L. E. B. 1995, A\&A, 300, 369
Aalto, S., Polatidis, A. G., Hüttemeister, S., \& Curran, S. J. 2002, A\&A, 381, 783

Aalto, S., Spaans, M., Wiedner, M. C., \& Hüttemeister, S. 2007, A\&A, 464, 193 Aalto, S., Wilner, D., Spaans, M., et al. 2009, A\&A, 493, 481

Baan, W. A., Henkel, C., Loenen, A. F., Baudry, A., \& Wiklind, T. 2008, A\&A, 477, 747

Baan, W. A., Loenen, A. F., \& Spaans, M. 2010, A\&A, 516, A40

Barger, A. J., Cowie, L. L., \& Sanders, D. B. 1999, ApJ, 518, L5

Bayet, E., Viti, S., Williams, D. A., \& Rawlings, J. M. C. 2008, ApJ, 676, 978

Blake, G. A., Sutton, E. C., Masson, C. R., \& Phillips, T. G. 1987, ApJ, 315, 621

Brown, J. M., Hougen, J. T., Huber, K. P., et al. 1975, J. Mol. Spect., 55, 500

Costagliola, F., \& Aalto, S. 2010, A\&A, 515, A71

de Vicente, P., Martín-Pintado, J., Neri, R., \& Colom, P. 2000, A\&A, 361, 1058

Evans, A. S., Becklin, E. E., Scoville, N. Z., et al. 2003, AJ, 125, 2341

Fuente, A., Martín-Pintado, J., Cernicharo, J., \& Bachiller, R. 1993, A\&A, 276, 473

Genzel, R., Lutz, D., Sturm, E., et al. 1998, ApJ, 498, 579

Gordon, M. A., Baars, J. W. M., \& Cocke, W. J. 1992, A\&A, 264, 337

Graciá-Carpio, J., García-Burillo, S., Planesas, P., \& Colina, L. 2006, ApJ, 640, L135

Greaves, J. S., \& Nyman, L. 1996, A\&A, 305, 950

Hirota, T., Yamamoto, S., Mikami, H., \& Ohishi, M. 1998, ApJ, 503, 717

Imanishi, M., Nakanishi, K., Kuno, N., \& Kohno, K. 2004, AJ, 128, 2037

Imanishi, M., Nakanishi, K., Tamura, Y., Oi, N., \& Kohno, K. 2007, AJ, 134, 2366

Jørgensen, J. K., Hogerheijde, M. R., Blake, G. A., et al. 2004, A\&A, 415, 1021

Joulain, K., Falgarone, E., Des Forets, G. P., \& Flower, D. 1998, A\&A, 340, 241

Kohno, K., Matsushita, S., Vila-Vilaró, B., et al. 2001, in The Central Kiloparsec of Starbursts and AGN: The La Palma Connection, ed. J. H. Knapen, J. E. Beckman, I. Shlosman, \& T. J. Mahoney, ASP Conf. Ser., 249, 672

Krips, M., Neri, R., García-Burillo, S., et al. 2008, ApJ, 677, 262

Lahuis, F., Spoon, H. W. W., Tielens, A. G. G. M., et al. 2007, ApJ, 659, 296

Lepp, S., \& Dalgarno, A. 1996, A\&A, 306,

Loenen, A. F., Spaans, M., Baan, W. A., \& Meijerink, R. 2008, A\&A, 488, L5

Lutz, D., Spoon, H. W. W., Rigopoulou, D., Moorwood, A. F. M., \& Genzel, R. 1998, ApJ, 505, L103

Maloney, P. R., Hollenbach, D. J., \& Tielens, A. G. G. M. 1996, ApJ, 466, 561

Martín, S., Mauersberger, R., Martín-Pintado, J., Henkel, C., \& García-Burillo, S. 2006, ApJS, 164, 450

Martín, S., Martín-Pintado, J., \& Mauersberger, R. 2009, ApJ, 694, 610

Martín, S., Aladro, R., Martín-Pintado, J., \& Mauersberger, R. 2010, A\&A, 522, A62

Mauersberger, R., \& Henkel, C. 1993, in ed. G. Klare, Rev. Mod. Astron., 6, 69

Meier, D. S., \& Turner, J. L. 2005, ApJ, 618, 259

Meijerink, R., \& Spaans, M. 2005, A\&A, 436, 397

Meijerink, R., Spaans, M., \& Israel, F. P. 2007, A\&A, 461, 793

Olsson, E., Aalto, S., Thomasson, M., \& Beswick, R. 2010, A\&A, 513, A11

Pérez-Beaupuits, J. P., Aalto, S., \& Gerebro, H. 2007, A\&A, 476, 177

Rodriguez-Franco, A., Martín-Pintado, J., \& Fuente, A. 1998, A\&A, 329, 1097

Sakamoto, K., Aalto, S., Wilner, D. J., et al. 2009, ApJ, 700, L104

Sanders, D. B., \& Mirabel, I. F. 1996, ARA\&A, 34, 749

Schilke, P., Walmsley, C. M., Pineau Des Forets, G., et al. 1992, A\&A, 256, 595

Solomon, P. M., Downes, D., Radford, S. J. E., \& Barrett, J. W. 1997, ApJ, 478, 144

Spoon, H. W. W., Marshall, J. A., Houck, J. R., et al. 2007, ApJ, 654, L49

Turner, B. E., Lee, H., \& Herbst, E. 1998, ApJS, 115, 91

Ungerechts, H., Bergin, E. A., Goldsmith, P. F., et al. 1997, ApJ, 482, 245

Veilleux, S., \& Osterbrock, D. E. 1987, ApJS, 63, 295

Wang, M., Henkel, C., Chin, Y., et al. 2004, A\&A, 422, 883

Wilson, T. L., \& Rood, R. 1994, ARA\&A, 32, 191

Wyrowski, F., Schilke, P., \& Walmsley, C. M. 1999, A\&A, 341, 882

Young, J. S., \& Sanders, D. B. 1986, ApJ, 302, 680 


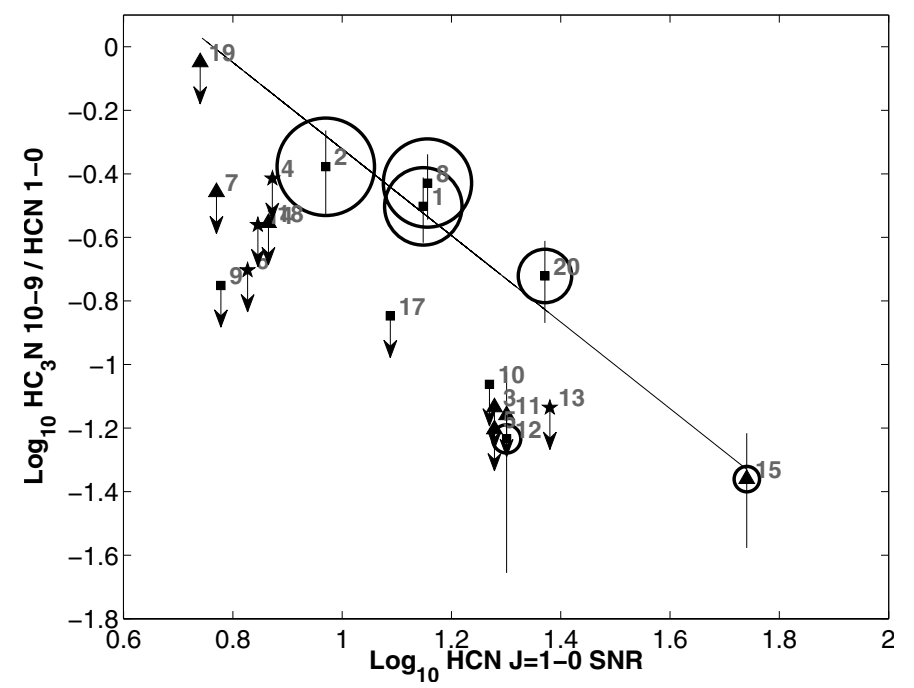

Fig. A.1. Ratio of $\mathrm{HC}_{3} \mathrm{~N} J=10-9$ over $\mathrm{HCN} J=1-0$ integrated intensities against the HCN $J=1-0$ signal-to-noise ratio. The line shows a linear fit through the $\mathrm{HC}_{3} \mathrm{~N}$ detections. See Fig. 4 for an explanation of the symbols.

\section{Appendix A: Detection of $\mathrm{HC}_{3} \mathrm{~N}$}

The ratio of $\mathrm{HC}_{3} \mathrm{~N} J=10-9$ over $\mathrm{HCN} J=1-0$ integrated intensities is plotted in Fig. A.1 against the HCN $J=1-0$ signal to noise ratio (SNR). The graph clearly shows that the non-detections are not caused by the sensitivity limit of our observations. In a wide range of SNR values, detections and nondetections coexist.

The line in Fig. A.1 shows a first order fit through the $\mathrm{HC}_{3} \mathrm{~N}$ detections, excluding the low-SNR detection in NGC 7771 (number 12 on the graph). Our detection limits (arrows in the graph) are, on average, 3 times fainter than the expected intensity derived by the fit. This means that in most of the cases - this may not be true for NGC 2273, number 19 in the graph - we do not detect $\mathrm{HC}_{3} \mathrm{~N}$ not because of a lack of sensitivity, but because the emission is intrinsically faint. In NGC 1068 the molecular emission is about one order of magnitude brighter than in the other galaxies of the sample, therefore the $\mathrm{HC}_{3} \mathrm{~N}$ detection (with the lowest $\mathrm{HC}_{3} \mathrm{~N} / \mathrm{HCN}$ ratio in the sample) in this galaxy may be only caused by its intrinsic high luminosity.

\section{Appendix B: $\mathrm{HCO}^{+}, \mathrm{HNC}$ and PAHs}

The strong dependence of the $\mathrm{HCO}^{+} / \mathrm{HNC}$ ratio with $\mathrm{PAH}$ equivalent width, shown in Fig. $4 d$, can be better understood by plotting the behaviour of the single tracers with increasing PAH EW.

In Fig. B. 1 we plot the ratios $\mathrm{HCO}^{+} / \mathrm{HCN}$ and $\mathrm{HNC} / \mathrm{HCN}$ against the PAH strength. Evidently, at high PAH EWs we have a depletion of HNC (respect to HCN) and an enhancement of $\mathrm{HCO}^{+}$. This two trends both contribute to the fast decrease of the $\mathrm{HCO}^{+} / \mathrm{HNC}$ ratio seen in Fig. $4 d$. For a discussion, see text in Sect. 5.10.
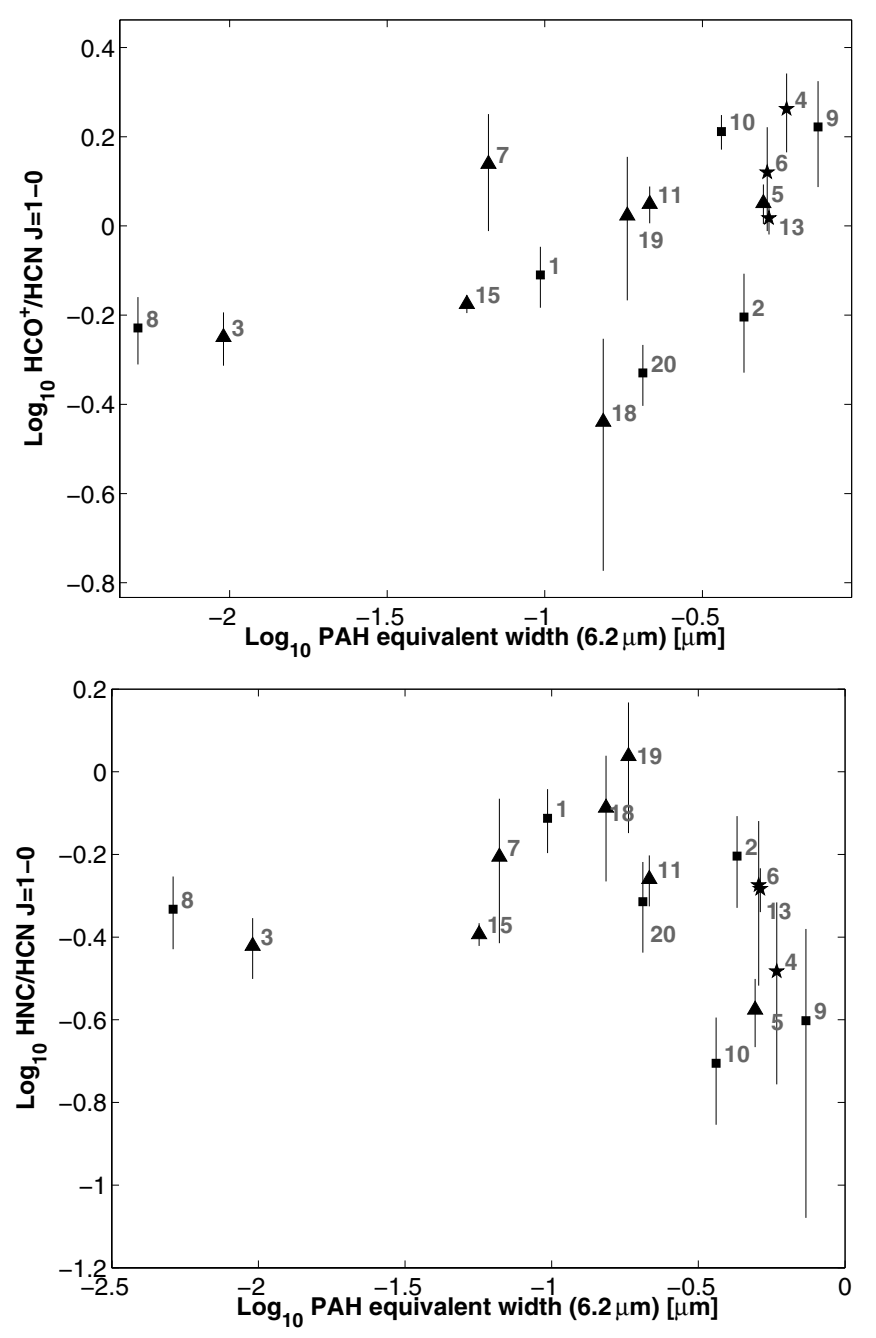

Fig. B.1. Line ratios of $\mathrm{HCO}^{+}$and $\mathrm{HNC}$ over $\mathrm{HCN}$ against the $\mathrm{PAH}$ equivalent width. Notice the decrease of $\mathrm{HNC}$ and increase of $\mathrm{HCO}^{+}$at high PAH EWs, to be compared with Fig. $4 d$.

\section{Appendix C: Comparison with the literature}

In Table C.1 we compare our observations with literature data from Baan et al. (2008). Antenna temperatures, obtained with EMIR, were translated into flux densities, by applying the $\mathrm{Jy} / \mathrm{K}$ conversion factor

$$
J[\mathrm{Jy}]=T_{\mathrm{A}}^{\star}[\mathrm{K}] \times 3.906 \times F_{\mathrm{eff}} / \eta_{\mathrm{A}},
$$

with $F_{\text {eff }}$ forward efficiency, and $\eta_{\mathrm{A}}$ antenna efficiency of the $30 \mathrm{~m}$ telescope at $88 \mathrm{GHz}$.

\section{Appendix D: EMIR Spectra}

In Figs. D.1-D.3 we show the spectra observed in all the galaxies in the sample. The intensities are in $T_{\mathrm{A}}^{\star}$, not corrected for beam efficiency (see Table 2). On the $x$-axis we plot the sky frequency in $\mathrm{GHz}$, not corrected for redshift. The position of the main emission lines is also shown.

\section{Appendix E: Derived line properties}


F. Costagliola et al.: EMIR molecular tracers

Table C.1. Comparison with data from the literature, as reported by Baan et al. (2008) (B08), and this work (EMIR). Exact references for the observations can be found in Table B.1 in Baan et al. (2008). Differences (in \%) between B08 and EMIR line intensity ratios are shown in column $\Delta \%$.

\begin{tabular}{|c|c|c|c|c|c|c|c|c|c|c|c|c|}
\hline \multirow[t]{2}{*}{ Galaxy } & \multicolumn{2}{|c|}{$\begin{array}{c}\mathrm{HCN} 1-0 \\
{\left[\mathrm{Jy} \mathrm{km} \mathrm{s}^{-1}\right]}\end{array}$} & \multicolumn{2}{|c|}{$\begin{array}{c}\text { HNC 1-0 } \\
{\left[\mathrm{Jy} \mathrm{km} \mathrm{s}^{-1}\right]}\end{array}$} & \multicolumn{2}{|c|}{$\begin{array}{l}\mathrm{HCO}^{+} 1-0 \\
{\left[\mathrm{Jy} \mathrm{km} \mathrm{s}^{-1}\right]} \\
\end{array}$} & \multicolumn{3}{|c|}{$\mathrm{HNC} / \mathrm{HCN}$} & \multicolumn{3}{|c|}{$\mathrm{HCO}^{+} / \mathrm{HCN}$} \\
\hline & B08 & EMIR & B08 & EMIR & B08 & EMIR & B08 & EMIR & $\Delta \%$ & B08 & EMIR & $\Delta \%$ \\
\hline NGC 1068 & 158 & 130 & 61.9 & 52.4 & 143 & 86.6 & 0.39 & 0.40 & 3 & 0.91 & 0.67 & 26 \\
\hline NGC 660 & 33 & 28.3 & 18.9 & 14.7 & 32.8 & 29.5 & 0.57 & 0.52 & 9 & 0.99 & 1.04 & 5 \\
\hline Mrk 231 & 17.6 & 11.2 & 12.1 & 4.24 & 12.6 & 6.3 & 0.69 & 0.38 & 45 & 0.72 & 0.56 & 21 \\
\hline NGC 6240 & 31.2 & 16.4 & 8 & 3.24 & 23.4 & 26.7 & 0.26 & 0.2 & 23 & 0.75 & 1.63 & 117 \\
\hline NGC 1614 & 7.2 & 4.83 & - & 1.59 & - & 8.84 & - & 0.33 & - & - & 1.83 & - \\
\hline UGC 5101 & 10.4 & 6.48 & - & 5.3 & - & 2.36 & - & 0.82 & - & - & 0.36 & - \\
\hline NGC 3079 & 43.1 & 24.6 & 32.3 & 6.54 & - & 27.7 & 0.75 & 0.27 & 65 & - & 1.12 & - \\
\hline NGC 3556 & 16.8 & 3.18 & - & 1.18 & - & 5.01 & - & 0.37 & - & - & 1.57 & - \\
\hline NGC 7469 & 10.8 & 11.8 & 17 & 6.48 & 15.8 & 13.2 & 1.57 & 0.55 & 65 & 1.46 & 1.12 & 23 \\
\hline NGC 7771 & 22.7 & 21.2 & - & 9.42 & 21.5 & 20 & - & 0.44 & - & 0.95 & 0.94 & 0.3 \\
\hline
\end{tabular}



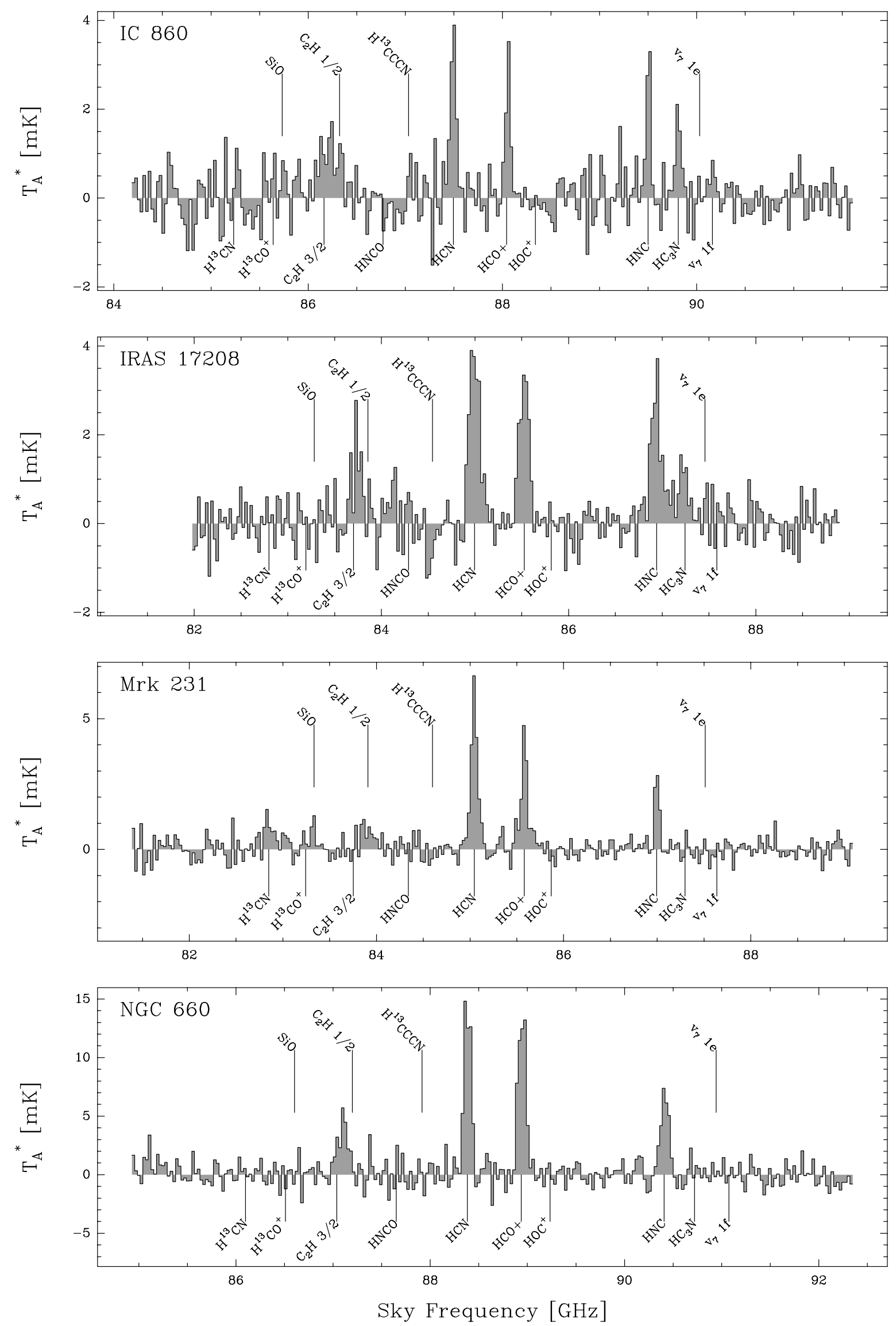

Fig. D.1. Observed spectra in the $88 \mathrm{GHz}$ band. The intensity scale is in $T_{\mathrm{A}}^{\star}$, not corrected for main beam efficiency. The main molecular transitions are labelled regardless of line detection. The $\mathrm{C}_{2} \mathrm{H} 3 / 2$ and $1 / 2$ labels mark the limits of the $\mathrm{C}_{2} \mathrm{H}$ multiplet at $87 \mathrm{GHz}$. Transitions of vibrationally excited $\mathrm{HC}_{3} \mathrm{~N}$ are labelled as $v_{7} 1 e$ and $v_{7} 1 f$. The frequency scale is the observed frequency, not corrected for redshift. 
F. Costagliola et al.: EMIR molecular tracers
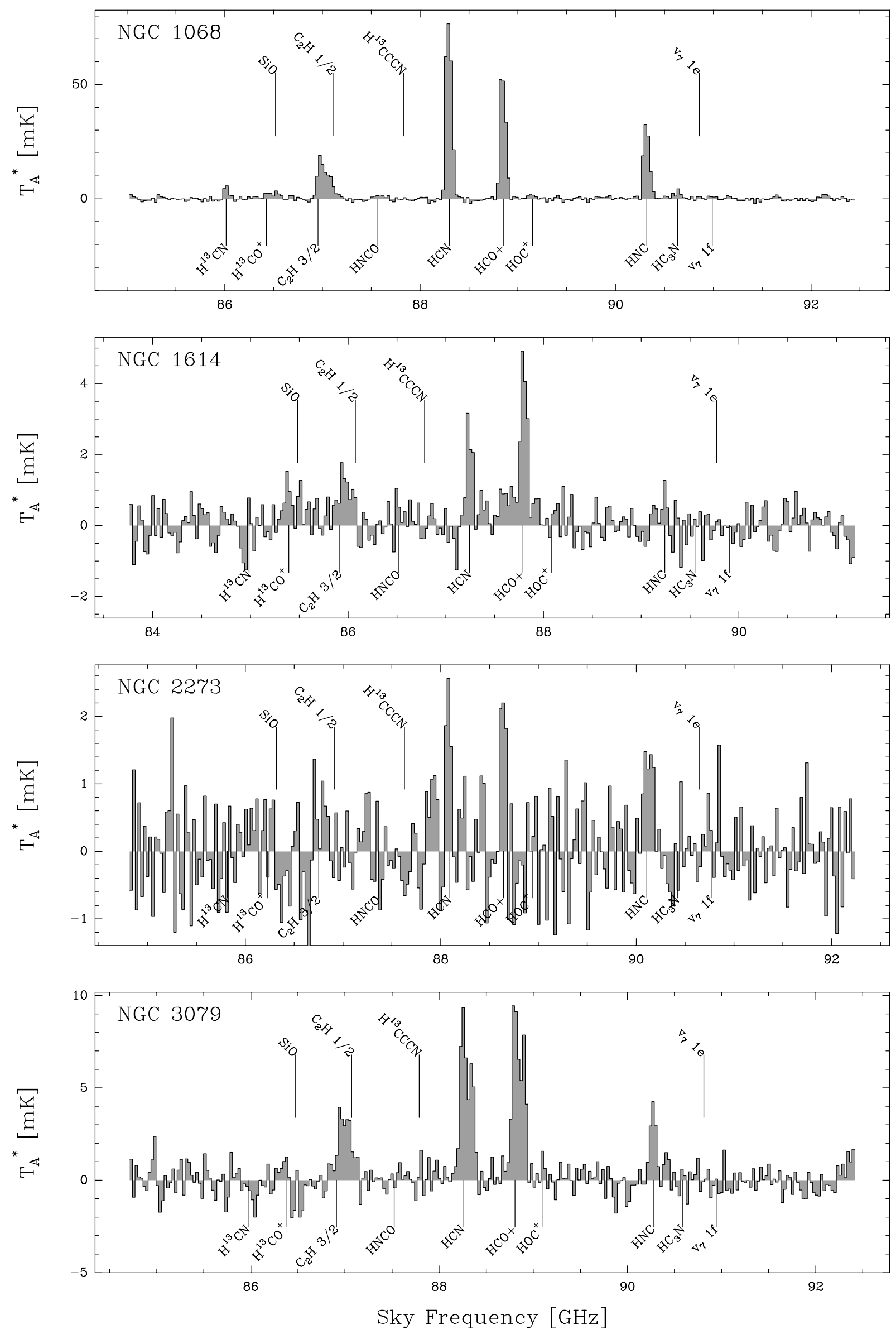

Fig. D.1. continued. 

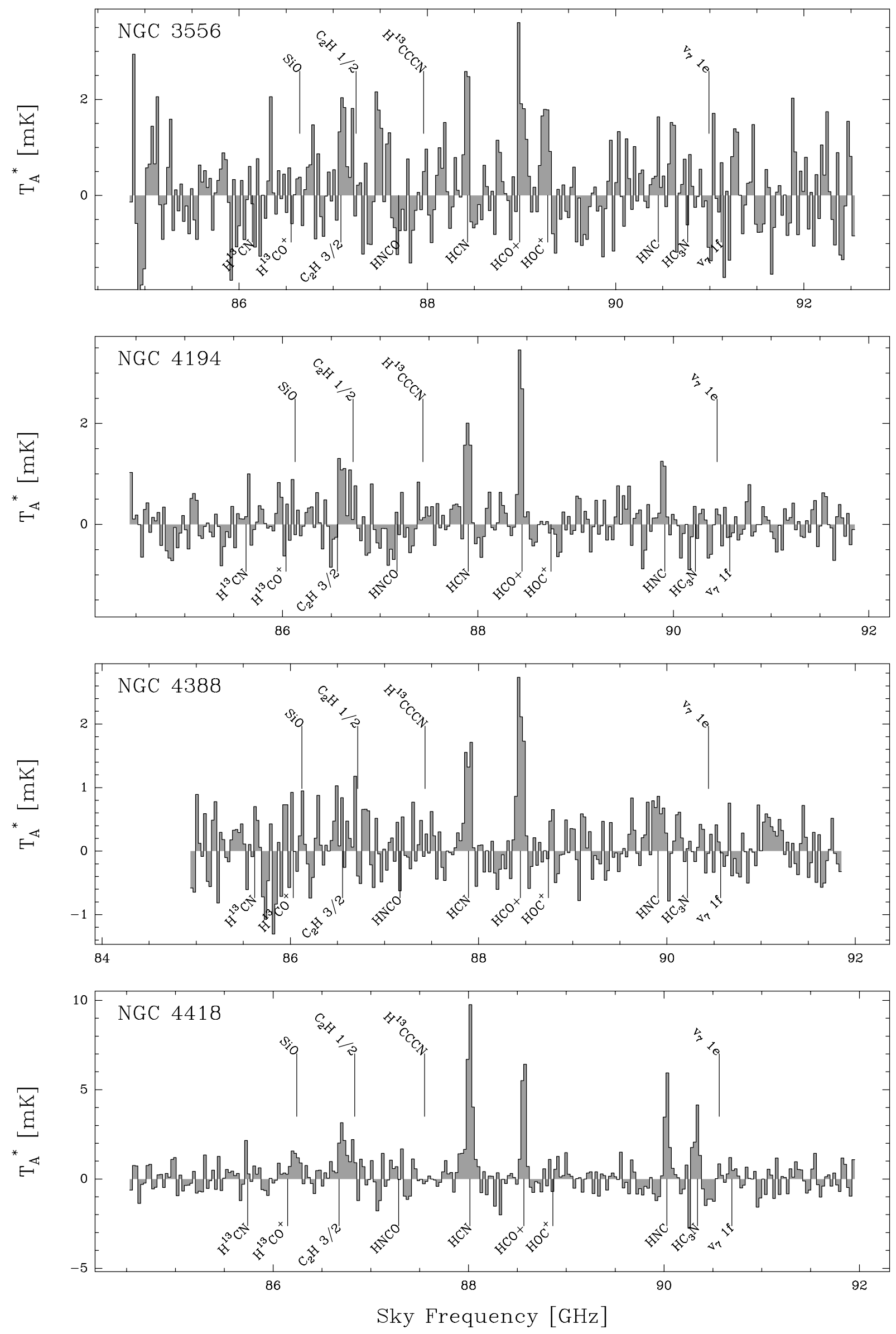

Fig. D.1. continued. 

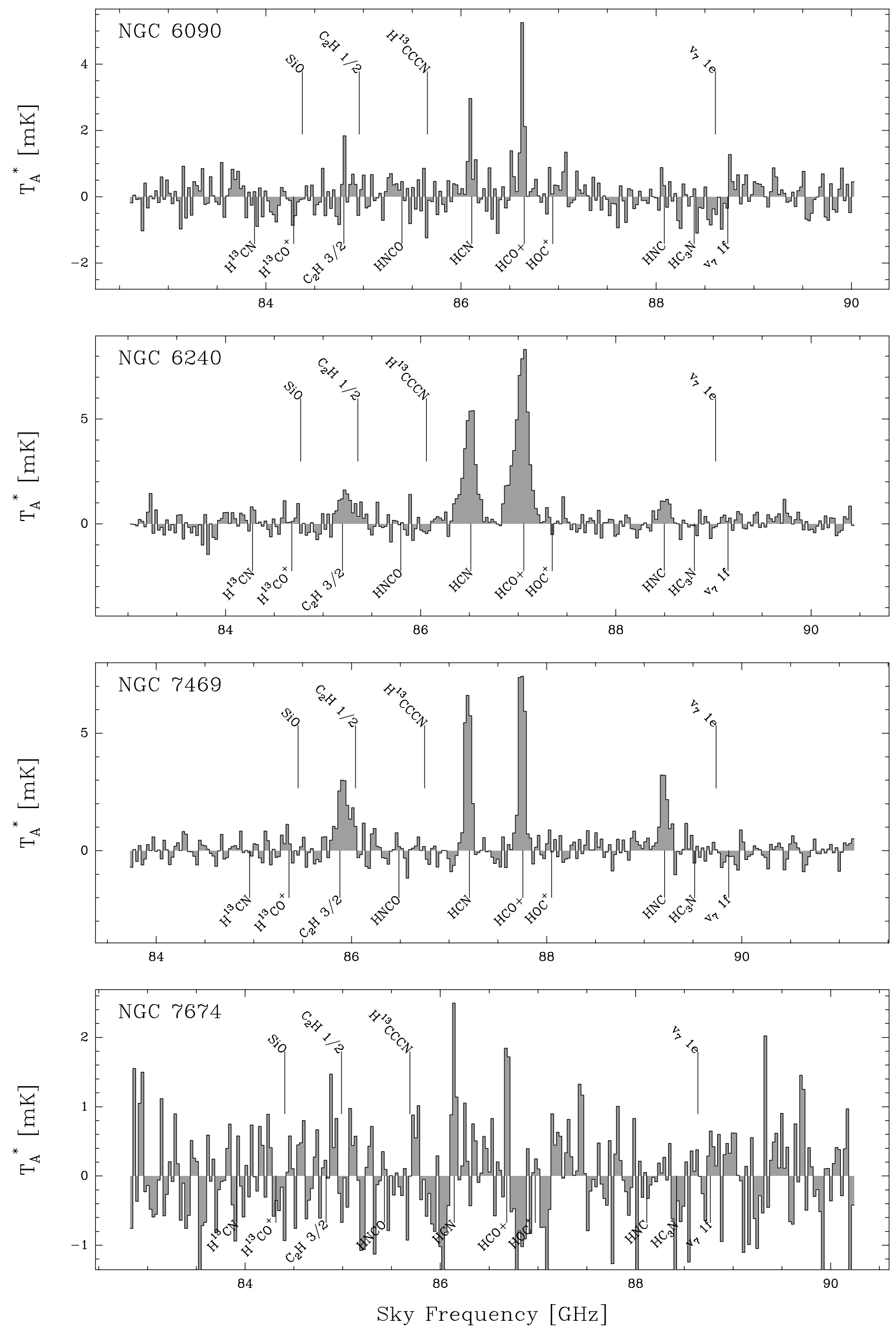

Fig. D.1. continued. 
A\&A 528, A30 (2011)
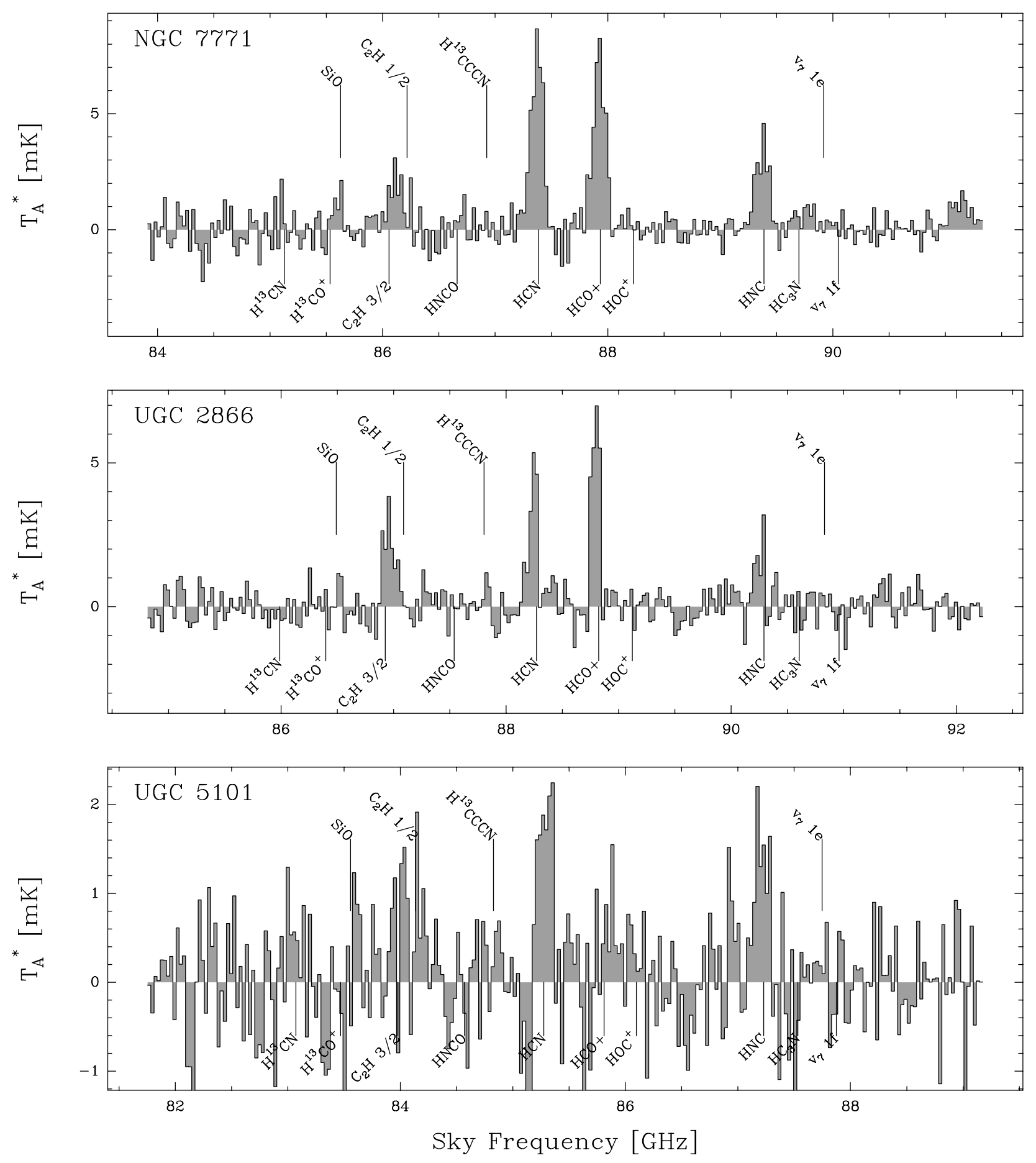

Fig. D.1. continued. 

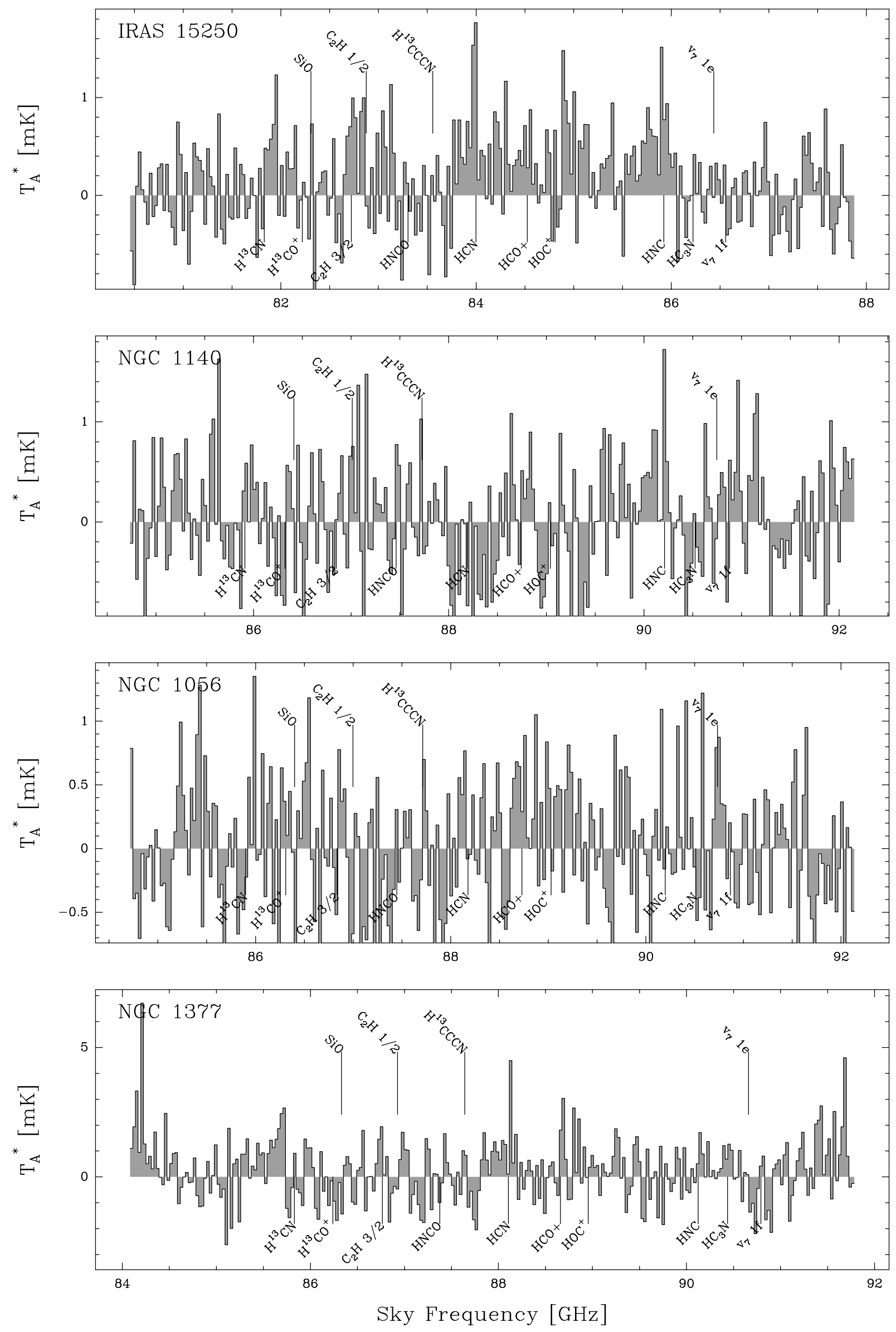

Fig. D.2. Non-detections in the $88 \mathrm{GHz}$ band. See caption of Fig. D.1. 

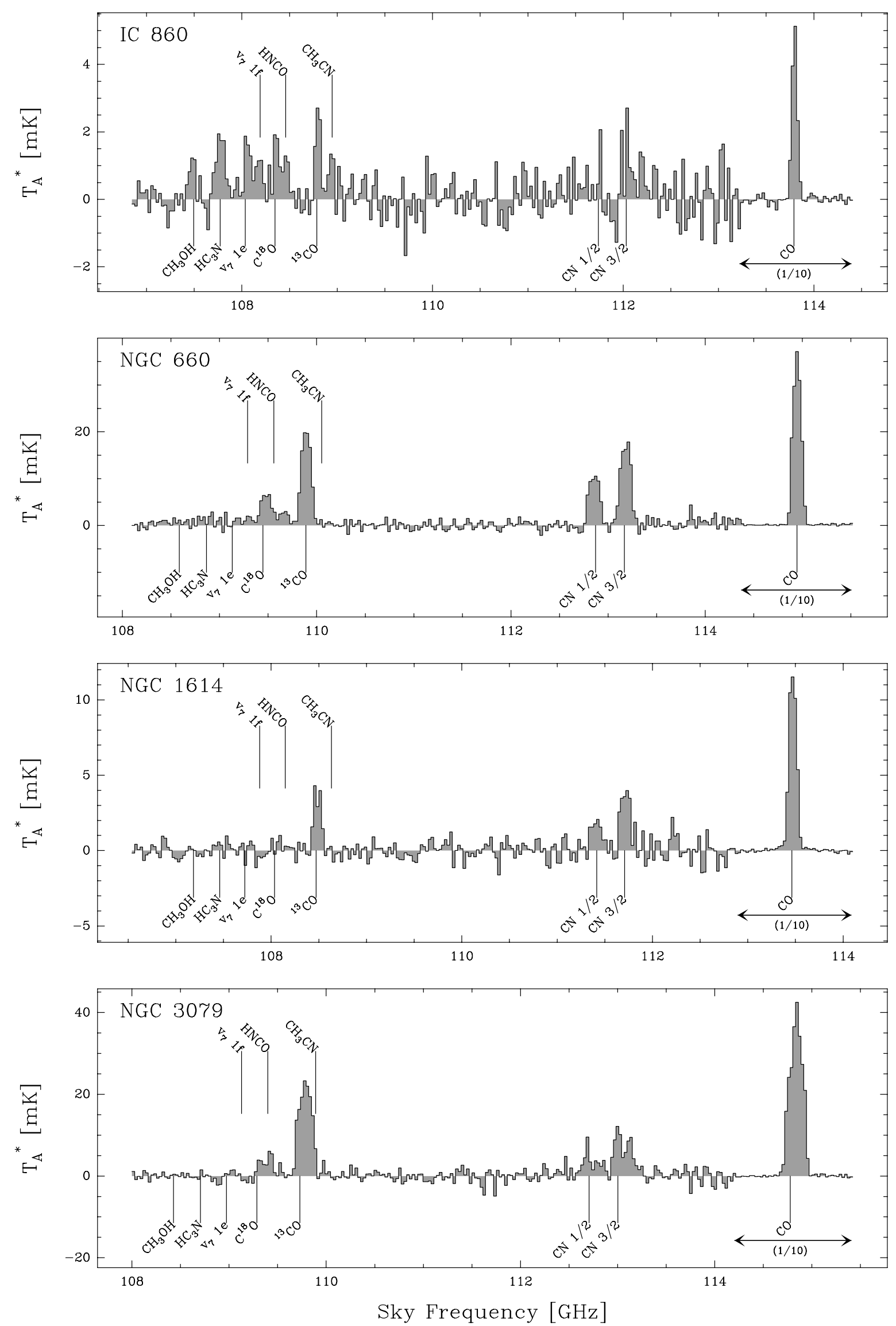

Fig. D.3. Observed spectra in the $112 \mathrm{GHz}$ band. The intensity scale is in $T_{\mathrm{A}}^{\star}$, not corrected for main beam efficiency. The region marked with $(1 / 10)$ around the CO 1-0 line was scaled down by a factor 10 . The main molecular transitions are labelled regardless of line detection. Transitions of vibrationally excited $\mathrm{HC}_{3} \mathrm{~N}$ are labelled as $v_{7} 1 e$ and $v_{7} 1 f$. The frequency scale is the observed frequency, not corrected for redshift. 
F. Costagliola et al.: EMIR molecular tracers
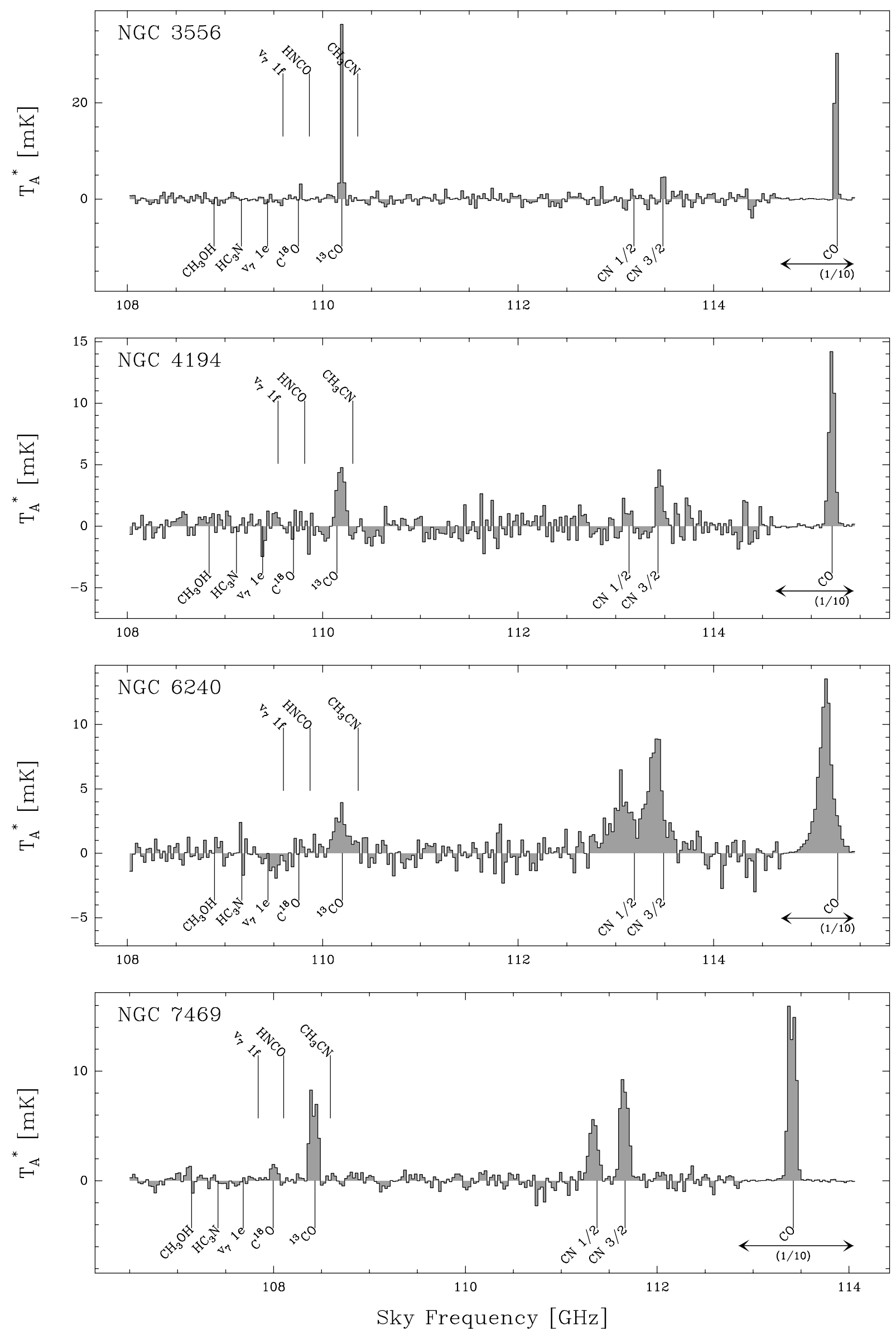

Fig. D.3. continued. 

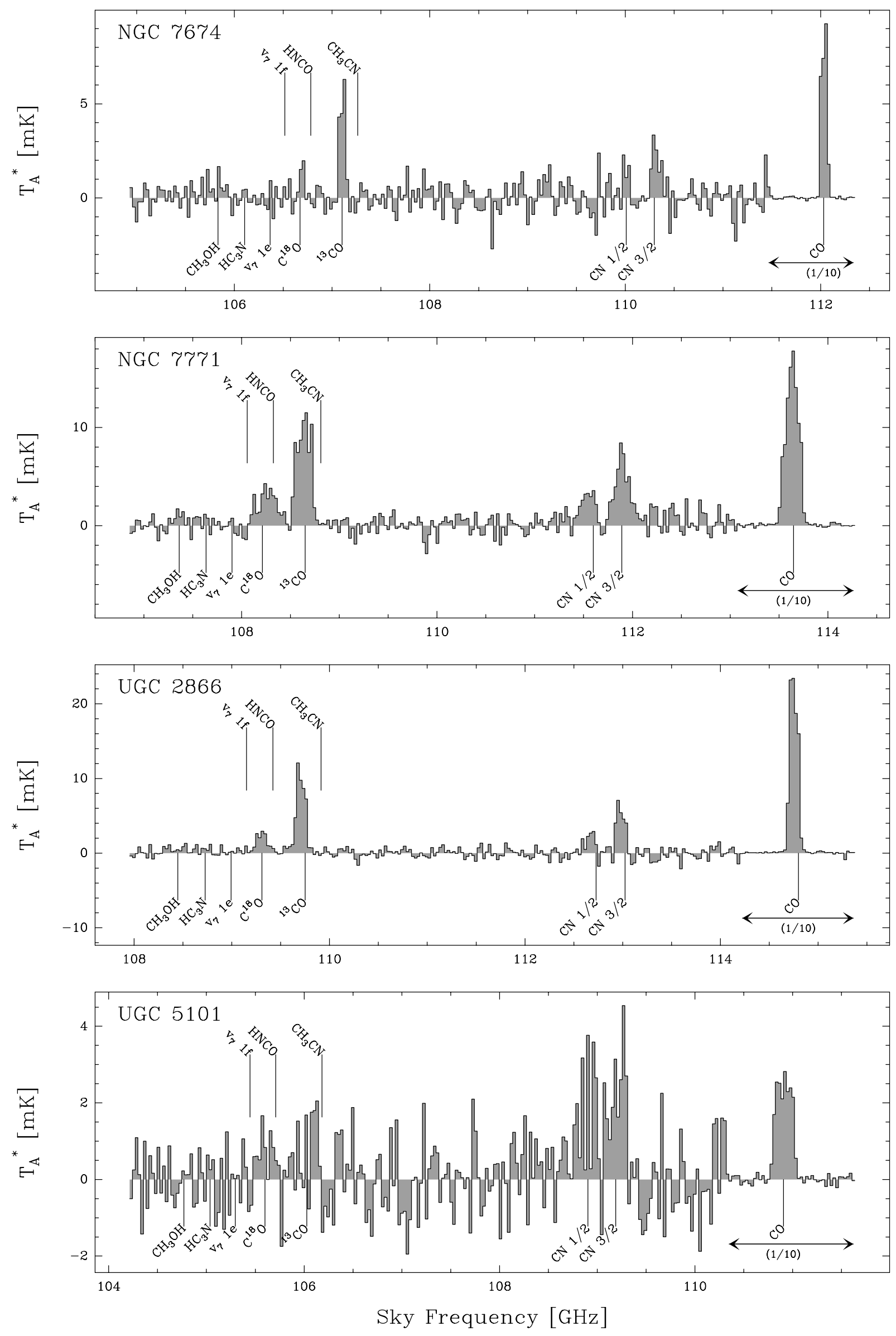

Fig. D.3. continued. 
Table E.1. Summary of the line properties of the observed galaxies. Integrated intensities and line widths were derived by means of Gaussian fitting. Uncertainties are shown in parenthesis.

\begin{tabular}{|c|c|c|c|c|c|c|}
\hline Galaxy & Transition & $\int_{\mathrm{K} \mathrm{km} \mathrm{s}^{-1}} T_{\mathrm{A}}^{\star} \mathrm{d} v$ & $\begin{array}{c}\Delta_{V} \\
\mathrm{~km} \mathrm{~s}^{-1}\end{array}$ & Transition & $\underset{\mathrm{K} \mathrm{km} \mathrm{s}^{-1}}{\int_{\mathrm{A}}^{\star} \mathrm{d} v}$ & $\begin{array}{l}\Delta_{V} \\
\mathrm{~s}^{-1} \\
\end{array}$ \\
\hline & $88 \mathrm{GHz}$ Band & & & $112 \mathrm{GHz}$ Band & & \\
\hline I 17208 & $\begin{array}{l}\mathrm{H} 13 \mathrm{CO}+1-0 \\
\text { SiO2-1 } \\
\text { H13CN1-0 } \\
\text { C2H1-0 } \\
\text { HCN1-0 } \\
\text { HCO+1-0 } \\
\text { HNC1-0 } \\
\text { HC3N10-9 }\end{array}$ & $\begin{array}{c}<2.55 \\
<0.23 \\
<0.23 \\
0.82(0.14) \\
1.97(0.14) \\
1.53(0.13) \\
1.52(0.16) \\
0.62(0.12)\end{array}$ & $\begin{array}{c}- \\
- \\
- \\
388(80)^{\star} \\
452(35) \\
407(38) \\
473(65) \\
452(70)\end{array}$ & & & \\
\hline IC 860 & $\begin{array}{l}\mathrm{H} 13 \mathrm{CO}+1-0 \\
\text { SiO2-1 } \\
\text { H13CN1-0 } \\
\text { C2H1-0 } \\
\text { HCN1-0 } \\
\text { HCO+1-0 } \\
\text { HNC1-0 } \\
\text { HC3N10-9 }\end{array}$ & $\begin{array}{c}<0.20 \\
<0.20 \\
<0.20 \\
1.86(0.36) \\
1.12(0.12) \\
0.70(0.10) \\
0.60(0.10) \\
0.43(0.09)\end{array}$ & $\begin{array}{c}- \\
- \\
- \\
1375(416)^{\star} \\
239(31) \\
202(38) \\
171(29) \\
200(67)\end{array}$ & $\begin{array}{l}\mathrm{CH} 3 \mathrm{OH} \\
\text { HC3N12-11 } \\
\text { HC3N12-11v7e } \\
\text { HC3N12-11v7f } \\
\text { C18O1-0 } \\
\text { 13CO1-0 } \\
\text { CN1-0J=1/2 } \\
\text { CN1-0J=3/2 } \\
\text { CO1-0 }\end{array}$ & $\begin{array}{c}0.40(0.13) \\
0.75(0.16) \\
0.33(0.08) \\
0.21(0.08) \\
0.50(0.20) \\
0.54(0.10) \\
<0.19 \\
<0.27 \\
10.24(0.17)\end{array}$ & $\begin{array}{c}312(104) \\
300(82) \\
170(60) \\
170(60) \\
253(150) \\
162(32) \\
- \\
- \\
179(3)\end{array}$ \\
\hline Mrk 231 & $\begin{array}{l}\mathrm{H} 13 \mathrm{CO}+1-0 \\
\mathrm{SiO} 2-1 \\
\text { H13CN1-0 } \\
\text { C2H1-0 } \\
\text { HCN1-0 } \\
\text { HCO+1-0 } \\
\text { HNC1-0 } \\
\text { HC3N10-9 }\end{array}$ & $\begin{array}{c}0.14(0.1) \\
0.25(0.11) \\
0.34(0.1) \\
0.38(0.20) \\
1.90(0.10) \\
1.07(0.09) \\
0.72(0.08) \\
<0.14\end{array}$ & $\begin{array}{c}271(62) \\
187(62) \\
228(68) \\
513(304)^{\star} \\
288(21) \\
208(23) \\
203(25) \\
-\end{array}$ & & & \\
\hline NGC 1614 & $\begin{array}{l}\mathrm{H} 13 \mathrm{CO}+1-0 \\
\mathrm{SiO} 2-1 \\
\mathrm{H} 13 \mathrm{CN} 1-0 \\
\mathrm{C} 2 \mathrm{H} 1-0 \\
\mathrm{HCN1} 1-0 \\
\mathrm{HCO}+1-0 \\
\mathrm{HNC} 1-0 \\
\text { HC3N10-9 }\end{array}$ & $\begin{array}{c}0.36(0.13) \\
0.24(0.13) \\
<0.28 \\
0.28(0.35) \\
0.79(0.29) \\
1.50(0.30) \\
0.24(0.23) \\
<0.28\end{array}$ & $\begin{array}{c}264(62) \\
270(62) \\
- \\
264(270)^{\star} \\
261(31) \\
290(75) \\
162(200) \\
-\end{array}$ & $\begin{array}{l}\mathrm{CH} 3 \mathrm{OH} \\
\text { HC3N12-11 } \\
\text { HC3N12-11v7e } \\
\text { C18O1-0 } \\
\text { 13CO1-0 } \\
\text { CN1-0J=1/2 } \\
\text { CN1-0J=3/2 } \\
\text { CO1-0 }\end{array}$ & $\begin{array}{c}<0.14 \\
0.14(0.14) \\
<0.14 \\
<0.14 \\
1.12(0.12) \\
0.62(0.13) \\
1.31(0.14) \\
33.71(0.15)\end{array}$ & $\begin{array}{c}- \\
264(237) \\
- \\
- \\
254(26) \\
305(58) \\
309(33) \\
267(2)\end{array}$ \\
\hline NGC 3079 & $\begin{array}{l}\mathrm{H} 13 \mathrm{CO}+1-0 \\
\mathrm{SiO} 2-1 \\
\mathrm{H} 13 \mathrm{CN} 1-0 \\
\text { C2H1-0 } \\
\text { HCN1-0 } \\
\text { HCO+1-0 } \\
\text { HNC1-0 } \\
\text { HC3N10-9 }\end{array}$ & $\begin{array}{l}0.33(0.14) \\
\quad<0.28 \\
<0.28 \\
2.26(0.24) \\
4.18(0.22) \\
4.70(0.23) \\
1.11(0.15) \\
<0.28\end{array}$ & $\begin{array}{c}250(40) \\
- \\
- \\
575(68)^{\star} \\
499(30) \\
516(27) \\
230(27) \\
-\end{array}$ & $\begin{array}{l}\mathrm{CH} 3 \mathrm{OH} \\
\text { HC3N12-11 } \\
\text { HC3N12-11v7e } \\
\text { C18O1-0 } \\
\text { 13CO1-0 } \\
\text { CN1-0J=1/2 } \\
\text { CN1-0J=3/2 } \\
\text { CO1-0 }\end{array}$ & $\begin{array}{c}<1.38 \\
<1.38 \\
<1.38 \\
2.00(0.20) \\
10.70(0.20) \\
0.88(0.40) \\
4.40(0.60) \\
183.00(9.00)\end{array}$ & $\begin{array}{c}- \\
- \\
- \\
254(16) \\
350(16) \\
85(16) \\
200(16) \\
200(16)\end{array}$ \\
\hline NGC 4194 & $\begin{array}{l}\mathrm{H} 13 \mathrm{CO}+1-0 \\
\text { SiO2-1 } \\
\text { H13CN1-0 } \\
\text { C2H1-0 } \\
\text { HCN1-0 } \\
\text { HCO+1-0 } \\
\text { HNC1-0 } \\
\text { HC3N10-9 }\end{array}$ & $\begin{array}{c}<0.09 \\
<0.09 \\
<0.09 \\
0.45(0.10) \\
0.47(0.07) \\
0.62(0.07) \\
0.25(0.07) \\
<0.09\end{array}$ & $\begin{array}{c}- \\
- \\
- \\
415(97)^{\star} \\
177(25) \\
151(19) \\
175(52) \\
-\end{array}$ & $\begin{array}{l}\mathrm{CH} 3 \mathrm{OH} \\
\text { HC3N12-11 } \\
\text { HC3N12-11v7e } \\
\text { C18O1-0 } \\
\text { 13CO1-0 } \\
\text { CN1-0J=1/2 } \\
\text { CN1-0J=3/2 } \\
\text { CO1-0 }\end{array}$ & $\begin{array}{c}<0.09 \\
<0.09 \\
<0.09 \\
0.09(0.20) \\
1.50(0.14) \\
0.50(0.14) \\
1.18(0.15) \\
28.65(0.17)\end{array}$ & $\begin{array}{c}- \\
- \\
- \\
220(30) \\
243(30) \\
200(30) \\
219(30) \\
180(30)\end{array}$ \\
\hline
\end{tabular}

Notes. $(\star)$ : For $\mathrm{C}_{2} \mathrm{H}$, the integrated intensity is obtained by fitting a single Gaussian to the multiplet at $87 \mathrm{GHz}$. This results in large line widths, which are not to be attributed to dynamics. 
Table E.1. continued.

\begin{tabular}{|c|c|c|c|c|c|c|}
\hline Galaxy & Transition & $\underset{\mathrm{K} \mathrm{km} \mathrm{s}^{-1}}{\int T_{\mathrm{A}}^{\star} \mathrm{d} v}$ & $\begin{array}{c}\Delta_{V} \\
\mathrm{~km} \mathrm{~s}^{-1}\end{array}$ & Transition & $\underset{\mathrm{K} \mathrm{km} \mathrm{s}^{-1}}{\int T_{\mathrm{A}}^{\star} \mathrm{d} v}$ & $\begin{array}{l}\Delta_{V} \\
\mathrm{~s}^{-1}\end{array}$ \\
\hline & $88 \mathrm{GHz}$ Band & & & $112 \mathrm{GHz}$ Band & & \\
\hline NGC 4388 & $\begin{array}{l}\mathrm{H} 13 \mathrm{CO}+1-0 \\
\mathrm{SiO} 2-1 \\
\mathrm{H} 13 \mathrm{CN} 1-0 \\
\mathrm{C} 2 \mathrm{H} 1-0 \\
\mathrm{HCN1}-0 \\
\mathrm{HCO}+1-0 \\
\mathrm{HNC1}-0 \\
\text { HC3N10-9 }\end{array}$ & $\begin{array}{c}<0.10 \\
<0.10 \\
<0.10 \\
0.10(0.20) \\
0.53(0.09) \\
0.73(0.09) \\
0.22(0.09) \\
<0.10\end{array}$ & $\begin{array}{c}- \\
- \\
- \\
225(541)^{\star} \\
228(50) \\
254(36) \\
192(93) \\
-\end{array}$ & & & \\
\hline NGC 4418 & $\begin{array}{l}\mathrm{H} 13 \mathrm{CO}+1-0 \\
\mathrm{SiO} 2-1 \\
\mathrm{H} 13 \mathrm{CN} 1-0 \\
\mathrm{C} 2 \mathrm{H} 1-0 \\
\mathrm{HCN} 1-0 \\
\mathrm{HCO}+1-0 \\
\mathrm{HNC1}-0 \\
\text { HC3N10-9 }\end{array}$ & $\begin{array}{c}<0.32 \\
0.32(0.14) \\
0.28(0.1) \\
0.32(0.28) \\
2.15(0.15) \\
1.27(0.13) \\
1.00(0.13) \\
0.80(0.13)\end{array}$ & $\begin{array}{c}- \\
150(33) \\
150(33) \\
148(33)^{\star} \\
188(33) \\
133(33) \\
150(33) \\
187(33)\end{array}$ & & & \\
\hline NGC 6090 & $\begin{array}{l}\mathrm{H} 13 \mathrm{CO}+1-0 \\
\mathrm{SiO} 2-1 \\
\mathrm{H} 13 \mathrm{CN} 1-0 \\
\mathrm{C} 2 \mathrm{H} 1-0 \\
\mathrm{HCN} 1-0 \\
\mathrm{HCO}+1-0 \\
\mathrm{HNC1}-0 \\
\text { HC3N10-9 }\end{array}$ & $\begin{array}{c}<0.09 \\
<0.09 \\
<0.09 \\
0.22(0.06) \\
0.48(0.08) \\
0.80(0.08) \\
0.12(0.06) \\
<0.09\end{array}$ & $\begin{array}{c}- \\
- \\
- \\
103(30)^{\star} \\
133(30) \\
128(30) \\
91(30) \\
-\end{array}$ & & & \\
\hline NGC 6240 & $\begin{array}{l}\mathrm{H} 13 \mathrm{CO}+1-0 \\
\mathrm{SiO} 2-1 \\
\mathrm{H} 13 \mathrm{CN} 1-0 \\
\mathrm{C} 2 \mathrm{H} 1-0 \\
\mathrm{HCN} 1-0 \\
\text { HCO+1-0 } \\
\text { HNC1-0 } \\
\text { HC3N10-9 }\end{array}$ & $\begin{array}{c}<0.24 \\
<0.24 \\
<0.24 \\
0.94(0.16) \\
2.79(0.15) \\
4.54(0.16) \\
0.55(0.13) \\
<0.24\end{array}$ & $\begin{array}{c}- \\
- \\
- \\
674(126)^{\star} \\
493(33) \\
541(24) \\
400(94) \\
-\end{array}$ & $\begin{array}{l}\mathrm{CH} 3 \mathrm{OH} \\
\text { HC3N12-11 } \\
\text { HC3N12-11v7e } \\
\text { C18O1-0 } \\
\text { 13CO1-0 } \\
\text { CN1-0J=1/2 } \\
\text { CN1-0J=3/2 } \\
\text { CO1-0 }\end{array}$ & $\begin{array}{c}<0.49 \\
<0.49 \\
<0.49 \\
1.00(0.18) \\
1.90(0.18) \\
2.00(0.20) \\
3.70(0.20) \\
54.70(0.50)\end{array}$ & $\begin{array}{c}- \\
- \\
- \\
400(70) \\
400(70) \\
400(70) \\
400(70) \\
414(70)\end{array}$ \\
\hline NGC 7469 & $\begin{array}{l}\mathrm{H} 13 \mathrm{CO}+1-0 \\
\mathrm{SiO} 2-1 \\
\mathrm{H} 13 \mathrm{CN} 1-0 \\
\mathrm{C} 2 \mathrm{H} 1-0 \\
\mathrm{HCN} 1-0 \\
\mathrm{HCO}+1-0 \\
\mathrm{HNC1}-0 \\
\text { HC3N10-9 }\end{array}$ & $\begin{array}{c}<0.14 \\
<0.14 \\
<0.14 \\
1.50(0.40) \\
1.97(0.10) \\
2.24(0.10) \\
1.10(0.10) \\
<0.14\end{array}$ & $\begin{array}{c}- \\
- \\
- \\
503(53)^{\star} \\
248(12) \\
346(11) \\
298(35) \\
-\end{array}$ & $\begin{array}{l}\mathrm{CH} 3 \mathrm{OH} \\
\text { HC3N12-11 } \\
\text { HC3N12-11v7e } \\
\text { C18O1-0 } \\
\text { 13CO1-0 } \\
\text { CN1-0J=1/2 } \\
\text { CN1-0J=3/2 } \\
\text { CO1-0 }\end{array}$ & $\begin{array}{c}0.16(0.14) \\
0.07(0.04) \\
<0.22 \\
0.33(0.07) \\
2.26(0.05) \\
1.80(0.12) \\
2.80(0.14) \\
47.00(0.02)\end{array}$ & $\begin{array}{c}55(407) \\
56(258) \\
- \\
209(41) \\
268(8) \\
298(10) \\
278(14) \\
274(1)\end{array}$ \\
\hline NGC 7771 & $\begin{array}{l}\mathrm{H} 13 \mathrm{CO}+1-0 \\
\mathrm{SiO} 2-1 \\
\mathrm{H} 13 \mathrm{CN} 1-0 \\
\text { C2H1-0 } \\
\text { HCN1-0 } \\
\text { HCO+1-0 } \\
\text { HNC1-0 } \\
\text { HC3N10-9 }\end{array}$ & $\begin{array}{c}<0.29 \\
0.23(0.36) \\
<0.29 \\
0.90(0.15) \\
3.40(0.15) \\
3.10(0.15) \\
2.00(0.16) \\
0.52(0.12)\end{array}$ & $\begin{array}{c}- \\
275(400) \\
- \\
442(78)^{\star} \\
396(19) \\
400(21) \\
469(44) \\
400(100)\end{array}$ & $\begin{array}{l}\mathrm{CH} 3 \mathrm{OH} \\
\text { HC3N12-11 } \\
\text { HC3N12-11v7e } \\
\text { C18O1-0 } \\
\text { 13CO1-0 } \\
\text { CN1-0J=1/2 } \\
\text { CN1-0J=3/2 } \\
\text { CO1-0 }\end{array}$ & $\begin{array}{c}0.44(0.14) \\
<0.42 \\
<0.42 \\
1.20(0.20) \\
5.60(0.20) \\
1.12(0.24) \\
2.78(0.26) \\
77.60(0.24)\end{array}$ & $\begin{array}{c}293(87) \\
- \\
- \\
445(81) \\
459(16) \\
400(30) \\
397(31) \\
422(2)\end{array}$ \\
\hline
\end{tabular}


Table E.1. continued.

\begin{tabular}{|c|c|c|c|c|c|c|}
\hline Galaxy & Transition & $\underset{\mathrm{K} \mathrm{km} \mathrm{s}^{-1}}{T_{\mathrm{A}}^{\star} \mathrm{d} v}$ & $\begin{array}{c}\Delta_{V} \\
\mathrm{~km} \mathrm{~s}^{-1}\end{array}$ & Transition & $\int_{\mathrm{K}} T_{\mathrm{A}}^{\star} \mathrm{d} v \mathrm{~s}^{-1}$ & $\begin{array}{l}\Delta_{V} \\
\mathrm{~s}^{-1}\end{array}$ \\
\hline & $88 \mathrm{GHz}$ Band & & & $112 \mathrm{GHz}$ Band & & \\
\hline NGC 660 & $\begin{array}{l}\mathrm{H} 13 \mathrm{CO}+1-0 \\
\text { SiO2-1 } \\
\text { H13CN1-0 } \\
\text { C2H1-0 } \\
\text { HCN1-0 } \\
\text { HCO+1-0 } \\
\text { HNC1-0 } \\
\text { HC3N10-9 }\end{array}$ & $\begin{array}{c}<0.35 \\
<0.35 \\
<0.35 \\
2.17(0.28) \\
4.80(0.20) \\
5.00(0.20) \\
2.50(0.20) \\
<0.35\end{array}$ & $\begin{array}{c}- \\
- \\
- \\
412(57)^{\star} \\
297(14) \\
318(16) \\
323(33) \\
-\end{array}$ & $\begin{array}{l}\mathrm{CH} 3 \mathrm{OH} \\
\text { HC3N12-11 } \\
\text { HC3N12-11v7e } \\
\text { C18O1-0 } \\
\text { 13CO1-0 } \\
\text { CN1-0J=1/2 } \\
\text { CN1-0J=3/2 } \\
\text { CO1-0 }\end{array}$ & $\begin{array}{c}<0.35 \\
<0.35 \\
<0.35 \\
2.42(0.22) \\
6.70(0.18) \\
4.10(0.18) \\
6.70(0.20) \\
110.20(0.30)\end{array}$ & $\begin{array}{c}- \\
- \\
- \\
361(54) \\
299(54) \\
334(54) \\
336(54) \\
271(54)\end{array}$ \\
\hline NGC3556 & $\begin{array}{l}\mathrm{H} 13 \mathrm{CO}+1-0 \\
\text { SiO2-1 } \\
\text { H13CN1-0 } \\
\text { C2H1-0 } \\
\text { HCN1-0 } \\
\text { HCO+1-0 } \\
\text { HNC1-0 } \\
\text { HC3N10-9 }\end{array}$ & $\begin{array}{c}<0.15 \\
<0.15 \\
<0.15 \\
0.84(0.08) \\
0.54(0.08) \\
0.85(0.08) \\
0.16(0.08) \\
<0.15\end{array}$ & $\begin{array}{c}- \\
- \\
- \\
409(66)^{\star} \\
73(66) \\
191(66) \\
81(66) \\
-\end{array}$ & $\begin{array}{l}\mathrm{CH} 3 \mathrm{OH} \\
\mathrm{HC} 3 \mathrm{~N} 12-11 \\
\text { HC3N12-11v7e } \\
\text { C18O1-0 } \\
\text { 13CO1-0 } \\
\mathrm{CN} 1-0 \mathrm{~J}=1 / 2 \\
\mathrm{CN} 1-0 \mathrm{~J}=3 / 2 \\
\mathrm{CO} 1-0\end{array}$ & $\begin{array}{c}<0.46 \\
<0.46 \\
<0.46 \\
0.26(0.05) \\
3.36(0.06) \\
0.40(0.10) \\
0.60(0.10) \\
42.00(0.30)\end{array}$ & $\begin{array}{c}- \\
- \\
- \\
54(5) \\
63(5) \\
103(5) \\
89(5) \\
80(5)\end{array}$ \\
\hline NGC1068 & $\begin{array}{l}\mathrm{H} 13 \mathrm{CO}+1-0 \\
\text { SiO2-1 } \\
\text { H13CN1-0 } \\
\text { C2H1-0 } \\
\text { HCN1-0 } \\
\text { HCO+1-0 } \\
\text { HNC1-0 } \\
\text { HC3N10-9 }\end{array}$ & $\begin{array}{c}0.6(0.5) \\
0.8(0.5) \\
1.26(0.5) \\
8.10(1.80) \\
22.00(0.40) \\
14.70(0.40) \\
8.90(0.40) \\
0.96(0.36)\end{array}$ & $\begin{array}{c}230(33) \\
230(33) \\
230(33) \\
471(33)^{\star} \\
241(33) \\
232(33) \\
238(33) \\
251(33)\end{array}$ & & & \\
\hline NGC 7674 & $\begin{array}{l}\mathrm{H} 13 \mathrm{CO}+1-0 \\
\text { SiO2-1 } \\
\text { H13CN1-0 } \\
\text { C2H1-0 } \\
\text { HCN1-0 } \\
\text { HCO+1-0 } \\
\text { HNC1-0 } \\
\text { HC3N10-9 }\end{array}$ & $\begin{array}{l}<0.18 \\
<0.18 \\
<0.18 \\
<0.18 \\
<0.18 \\
<0.18 \\
<0.18 \\
<0.18\end{array}$ & $\begin{array}{l}- \\
- \\
- \\
- \\
- \\
- \\
-\end{array}$ & $\begin{array}{l}\mathrm{CH} 3 \mathrm{OH} \\
\text { HC3N12-11 } \\
\text { HC3N12-11v7e } \\
\text { C18O1-0 } \\
\text { 13CO1-0 } \\
\text { CN1-0J=1/2 } \\
\text { CN1-0J=3/2 } \\
\text { CO1-0 }\end{array}$ & $\begin{array}{c}<0.51 \\
<0.51 \\
<0.51 \\
0.51(0.10) \\
1.40(0.10) \\
0.51(0.14) \\
1.10(0.20) \\
20.25(0.20)\end{array}$ & $\begin{array}{c}- \\
- \\
- \\
238(50) \\
196(13) \\
238(62) \\
317(67) \\
200(16)\end{array}$ \\
\hline UGC 2866 & $\begin{array}{l}\mathrm{H} 13 \mathrm{CO}+1-0 \\
\text { SiO2-1 } \\
\mathrm{H} 13 \mathrm{CN} 1-0 \\
\mathrm{C} 2 \mathrm{H} 1-0 \\
\mathrm{HCN1}-0 \\
\mathrm{HCO}+1-0 \\
\mathrm{HNC1}-0 \\
\text { HC3N10-9 }\end{array}$ & $\begin{array}{c}<0.21 \\
<0.21 \\
<0.21 \\
1.30(0.15) \\
1.47(0.12) \\
2.15(0.12) \\
0.75(0.13) \\
<0.21\end{array}$ & $\begin{array}{c}- \\
- \\
- \\
397(50)^{\star} \\
254(25) \\
273(15) \\
336(55) \\
-\end{array}$ & $\begin{array}{l}\mathrm{CH} 3 \mathrm{OH} \\
\text { HC3N12-11 } \\
\text { HC3N12-11v7e } \\
\text { C18O1-0 } \\
\text { 13CO1-0 } \\
\text { CN1-0J=1/2 } \\
\text { CN1-0J=3/2 } \\
\text { CO1-0 }\end{array}$ & $\begin{array}{c}<0.60 \\
<0.60 \\
<0.60 \\
0.60(0.14) \\
3.46(0.12) \\
0.69(0.12) \\
1.98(0.16) \\
71.54(0.28)\end{array}$ & $\begin{array}{c}- \\
- \\
- \\
283(56) \\
283(10) \\
190(30) \\
264(22) \\
266(1)\end{array}$ \\
\hline UGC 5101 & $\begin{array}{l}\mathrm{H} 13 \mathrm{CO}+1-0 \\
\text { SiO2-1 } \\
\text { H13CN1-0 } \\
\text { C2H1-0 } \\
\text { HCN1-0 } \\
\text { HCO+1-0 } \\
\text { HNC1-0 } \\
\text { HC3N10-9 }\end{array}$ & $\begin{array}{c}<0.31 \\
<0.31 \\
0.37(0.17) \\
0.98(0.27) \\
1.10(0.15) \\
0.40(0.16) \\
0.90(0.18) \\
<0.31\end{array}$ & $\begin{array}{c}- \\
- \\
456(130) \\
1043(130)^{\star} \\
455(130) \\
500(130) \\
476(130) \\
-\end{array}$ & $\begin{array}{l}\mathrm{CH} 3 \mathrm{OH} \\
\text { HC3N12-11 } \\
\text { HC3N12-11v7e } \\
\text { C18O1-0 } \\
\text { 13CO1-0 } \\
\text { CN1-0J=1/2 } \\
\text { CN1-0J=3/2 } \\
\text { CO1-0 }\end{array}$ & $\begin{array}{c}<1.85 \\
<1.85 \\
<1.85 \\
<1.85 \\
<1.85 \\
1.85(0.19) \\
1.85(0.19) \\
16.20(0.30)\end{array}$ & $\begin{array}{c}- \\
- \\
- \\
- \\
- \\
578(112) \\
578(112) \\
536(112)\end{array}$ \\
\hline NGC 2273 & $\begin{array}{l}\mathrm{H} 13 \mathrm{CO}+1-0 \\
\mathrm{SiO} 2-1 \\
\mathrm{H} 13 \mathrm{CN} 1-0 \\
\mathrm{C} 2 \mathrm{H} 1-0 \\
\mathrm{HCN} 1-0 \\
\text { HCO+1-0 } \\
\text { HNC1-0 } \\
\text { HC3N10-9 }\end{array}$ & $\begin{array}{c}<0.33 \\
<0.33 \\
<0.33 \\
<0.33 \\
0.55(0.10) \\
0.33(0.10) \\
0.33(0.10) \\
<0.33\end{array}$ & $\begin{array}{c}- \\
- \\
- \\
- \\
154(66) \\
154(66) \\
154(66) \\
-\end{array}$ & & & \\
\hline
\end{tabular}


A\&A 528, A30 (2011)

Table E.2. Upper limits (3- $\sigma$ ) for non-detections, estimated from noise $r m s$. The assumed line width was derived from CO 1-0 observations.

\begin{tabular}{|c|c|c|c|c|c|}
\hline Galaxy & Transition & $\underset{\mathrm{K} \mathrm{km} \mathrm{s}}{\int T_{\mathrm{A}}^{\star} \mathrm{d} v}$ & $\begin{array}{c}\Delta_{V} \\
\mathrm{~km} \mathrm{~s}^{-1}\end{array}$ & $\underset{\mathrm{K} \mathrm{km} \mathrm{s}^{-1}}{T_{\mathrm{A}}^{\star} \mathrm{d} v}$ & $\begin{array}{l}\Delta_{V} \\
\mathrm{~s}^{-1}\end{array}$ \\
\hline & $88 \mathrm{GHz}$ Band & & & $112 \mathrm{GHz}$ Band & \\
\hline NGC 1377 & - & $<0.3$ & 70 & & \\
\hline IRAS 15250 & - & $<2.7$ & 170 & & \\
\hline
\end{tabular}

